\title{
Engineering the crack path by controlling the microstructure
}

\author{
A. Srivastava ${ }^{\mathrm{a}}$, S. Osovski ${ }^{\mathrm{b}}$, A. Needleman ${ }^{\mathrm{a}, *}$ \\ ${ }^{a}$ Department of Materials Science and Engineering, Texas A $8 M$ University, College Station, TX, USA \\ ${ }^{b}$ Faculty of Mechanical Engineering, Technion - Israel Institute of Technology, Haifa, Israel
}

\begin{abstract}
We explore the possibility of engineering the crack path by controlling a material's microstructure in order to increase its crack growth resistance. Attention is confined to a specific type of microstructure that is encountered in a variety of structural metals and alloys - second phase particles distributed in a ductile matrix. The type of controlled microstructure modeled is characterized by various sinusoidal distributions of particles with fixed mean particle spacing. Three dimensional, finite deformation small scale yielding calculations of mode I crack growth are carried out for such controlled microstructures using an elastic-viscoplastic constitutive relation for a progressively cavitating solid. The results show that appropriately engineered sinusoidal distributions of particles can give fracture toughness values 2 to 3 times greater than a random distribution of particles with the same mean particle spacing. Tearing modulus values can be increased by a factor of 1.5 to 2 . The greatest crack growth resistance generally occurs when the amplitude and the wavelength of the sinusoidal distribution are increased together. When the amplitude and the wavelength of the sinusoidal distribution do not increase together the crack can jump from one crest (or trough) to the next crest (or trough) which tends to reduce the crack growth resistance. Fracture surface roughness statistics are also calculated. In contrast to the essentially universal value for random distributions of particles, the value of the computed Hurst exponent is found to depend on the amplitude and the wavelength of the sinusoidal profile. A correlation is found between the computed fracture toughness values and values of characteristic length scales of the fracture surface roughness.
\end{abstract}

Keywords: Ductile fracture, Fracture surface roughness, Micromechanical modeling, Finite elements, Crack path engineering

\section{Introduction}

Ductile fracture limits the performance, safety, reliability and manufacturability of a variety of engineering components and structures; for example, the crash worthiness of automobiles, the integrity of pipelines, the blast resistance of ships and airplane cargo holds,

Email address: needle@tamu.edu (A. Needleman)

Preprint submitted to Elsevier

October 19, 2016 
and the manufacturability of sheet metal components. The mechanism of room temperature ductile fracture in engineering metals and alloys involves the nucleation, growth and coalescence of micron scale voids, Tipper (1949). This mechanism leads to the relatively high crack growth resistance associated with ductile fracture. To quantify this, we note that the atomic work of separation for any material is in the range $1-10 \mathrm{~J} / \mathrm{m}^{2}$ see, for example, Hayes et al. (2004) and Van der Ven and Ceder (2004) while the work per unit area for ductile crack growth in a metal is typically in the range $10^{4}-10^{5} \mathrm{~J} / \mathrm{m}^{2}$, e.g. Lautridou and Pineau (1981). The difference comes from micron and larger scale plastic dissipation. This implies that: (i) key processes for ductile fracture occur at a scale that can be modeled using continuum mechanics; and (ii) changes in the material microstructure that lead to increased dissipation can have a major effect on crack growth resistance. Reviews from various perspective of the mechanics and physics of ductile fracture by porosity evolution have been given by Goods and Brown (1979); Garrison and Moody (1987); Tvergaard (1990); Benzerga and Leblond (2010); Pineau et al. (2016); Benzerga et al. (2016).

Recent computational analyses have shown that ductile crack growth resistance is strongly affected by the crack path (Srivastava et al., 2014; Osovski et al., 2015a,b). Following these, we explore the possibility of engineering the crack path by controlling a material's microstructure in order to increase the material's crack growth resistance. Attention is confined to circumstances where, for ductile fracture, the dominant feature of the microstructure is a distribution of second phase particles in a ductile material matrix, as is encountered in a variety of ferrous and aluminum alloys. The voids nucleate either by debonding or cracking of second phase particles and/or inclusions (Puttick, 1959; Rogers, 1960; Gurland and Plateau, 1963). These second phase particles are either added intentionally to the material's microstructure (for precipitation-hardening or dispersion-strengthening as in ferrous and aluminum alloys, Martin (1998)) or their formation is an unavoidable consequence of processing.

In the past few decades there have been rapid advances in inverse design methodologies that facilitate material design to achieve a targeted performance metric, Olson (1997); McDowell (2007); McDowell and Olson (2009); Fullwood et al. (2010). But there are relatively few studies aimed at designing more fracture resistant ductile materials (Hao et al., 2004, 2003; Kulkarni et al., 2004). In the work of Hao et al. (2004, 2003) the focus was on the early stages of ductile crack growth in steels and the effects of microstructural heterogeneities. Also, in those studies the material's microstructure entered the design space in a homogenized form, i.e. the spatial variation of the microstructure were either smeared out or defined in terms of correlation functions. However, with the emergence of manufacturing technologies such as additive manufacturing, Herderick (20011); Frazier (2014), it is becoming possible to control the microstructural features of a metallic alloy. In addition, the emergence of 3D tomography techniques (Uchic et al., 2007; Ueda et al., 2014; Kahziz et al., 2016) make it possible to characterize the three dimensional microstructural features. The capability to characterize and control microstructural features provides the motivation for developing a predictive computational framework relating material microstructure and ductile crack growth resistance. Such a framework will enable the exploration of bounds for fracture toughness which is largely set by the crack path. 
Microstructures with randomly distributed second phase void nucleating particles have been modeled in a variety of two dimensional and three dimensional ductile fracture analyses, e.g. Needleman and Tvergaard (1987); Mathur et al. (1996); Tvergaard and Needleman (2006); Needleman et al. (2012); Ponson et al. (2013); Tang et al. (2013); Srivastava et al. (2014); Osovski et al. (2015a). In particular, in Srivastava et al. (2014); Osovski et al. (2015a), ductile crack growth in such microstructures were computed to a sufficient extent and in sufficient detail so as to be able to characterize the crack growth resistance in terms of both $J_{I C}$ and the non-dimensional tearing modulus $T_{R}$, as well as to quantify the fracture surface roughness statistics. In certain circumstances, a quantitative relation was found between a parameter characterizing the random microstructure, a mean particle spacing, and parameters characterizing both the ductile crack growth resistance and the fracture surface roughness. Together these gave a quantitative relation between measures of fracture surface roughness and of ductile crack growth resistance. However, for the random particle distributions considered in Srivastava et al. (2014); Osovski et al. (2015a), it was not possible to identify the key controllable features of the particle distribution that act to set the crack path.

Here, we carry out analyses of ductile crack growth using the same framework as in Srivastava et al. (2014); Osovski et al. (2015a) but for controlled distributions of second phase void nucleating particles. In particular, the controlled void nucleating particle distributions considered are various sinusoidal distributions with specified mean particle spacings. The particles are constrained to lie along a sinusoidal profile but within the profile the particle distribution is random with a specified mean particle spacing. The three dimensional finite deformation finite element analyses of ductile crack growth are based on a constitutive framework for a progressively cavitating ductile solid stemming from the work of Gurson (1975). The calculations are carried out for small scale yielding conditions with monotonically increasing imposed remote displacements corresponding to mode I loading. We note that crack propagation along a continuous weak interface that follows a sinusoidal or wavy profile between two materials under dominant mode I loading has been studied, both computationally (Zavattieri et al., 2007, 2008; Cordisco et al., 2012, 2014) and experimentally (Mirkhalaf et al., 2014; Malik and Barthelat, 2016). These computational and experimental studies have shown that, in the circumstances considered, crack deflection along controlled wavy interfaces can improve crack growth resistance.

The aim of our calculations is threefold: (i) to investigate the range of values of measures of ductile crack growth resistance $\left(J_{I C}\right.$ and $\left.T_{R}\right)$ that can be achieved with a controlled distribution of void nucleating particles and compare these values with those obtained for a random distribution; (ii) to explore whether the results for controlled distributions can serve to isolate the key particle distribution features controlling ductile crack growth resistance, in order to indicate a path to microstructural design for improved ductile crack growth resistance; and (iii) to investigate the relation between quantitative measures of fracture surface roughness and crack growth resistance for a controlled distribution of void nucleating particles, so as to identify key observable fracture surface features that can be quantitatively related to crack growth resistance. In particular, in Srivastava et al. (2014), for a range of void nucleating particle densities, a linear relation was found between the 
crack growth resistance measures $J_{I C}$ and $T_{R}$ and a fracture surface roughness length scale. For a sinusoidal crack path, this length scale is directly related to the wavelength. Hence, by analyzing a variety of sinusoidal distributions the extent to which this relation holds can be investigated.

\section{Problem formulation and numerical method}

The initial/boundary value problem analyzed and the numerical implementation are same as in Needleman et al. (2012); Ponson et al. (2013); Srivastava et al. (2014); Osovski et al. $(2015 \mathrm{a}, \mathrm{b})$. Here we briefly describe the formulation for completeness, 8more complete descriptions are given in the references cited.

A mode I small scale yielding boundary value problem is analyzed for a slice of material with an initial through thickness crack as shown in Fig. 1. The finite element calculations are based on the dynamic principle of virtual work using a finite deformation Lagrangian convected coordinate formulation. Material points are referred to using a Cartesian coordinate system $(x, y, z)$ with positions in the initial configuration denoted as $\left(x_{0}, y_{0}, z_{0}\right)$. Displacements corresponding to the quasi-static mode I isotropic elastic singular displacement field are imposed on the remote boundaries of the region analyzed. Also, the initial velocity field in the region analyzed corresponds to that of the mode I singular field. These initial and boundary conditions aim at providing an approximation to quasi-static response. The slice of material analyzed has the dimensions $h_{x} \times h_{y} \times h_{z}$, where, $h_{x}=h_{y}=0.4 \mathrm{~m}$ and $h_{z} / h_{x}=0.0125$, with an initial crack tip of opening $b_{0}=1.875 \times 10^{-4} \mathrm{~m}$ and symmetry boundary conditions imposed on $z_{0}=0, h_{z}$ planes. For quasi-static response, only the ratio of geometric lengths matter, not their absolute magnitude. The finite element mesh consists of 428, 256 twenty node brick elements giving 1, 868, 230 nodes and 5, 604, 690 degrees of freedom. Ten uniformly spaced elements are used through the thickness $h_{z}$. A uniform $208 \times 64$ in-plane $\left(x_{0}-y_{0}\right.$ plane) mesh is used in a $0.02 \mathrm{~m} \times 0.006 \mathrm{~m}$ region (marked with dashed line in the schematic in Fig. 1) immediately in front of the initial crack tip with in-plane elements of dimension $9.62 \times 10^{-5} \mathrm{~m}$ by $9.38 \times 10^{-5} \mathrm{~m}$. The element dimension $e_{x}=9.62 \times 10^{-5} \mathrm{~m}$ serves as a normalization length.

The constitutive framework used here is the modified Gurson constitutive relation (for more details see Tvergaard (1990)) with the flow potential having the form (Gurson, 1975)

$$
\Phi=\frac{\sigma_{e}^{2}}{\bar{\sigma}^{2}}+2 q_{1} f^{*} \cosh \left(\frac{3 q_{2} \sigma_{h}}{2 \bar{\sigma}}\right)-1-\left(q_{1} f^{*}\right)^{2}=0
$$

where $q_{1}=1.25, q_{2}=1.0$ are parameters introduced in Tvergaard $(1981,1982 \mathrm{a}), f$ is the void volume fraction, $\bar{\sigma}$ is the matrix flow strength, and

$$
\sigma_{e}^{2}=\frac{3}{2} \boldsymbol{\sigma}^{\prime}: \boldsymbol{\sigma}^{\prime} \quad, \quad \sigma_{h}=\frac{1}{3} \boldsymbol{\sigma}: \mathbf{I} \quad, \quad \boldsymbol{\sigma}^{\prime}=\boldsymbol{\sigma}-\sigma_{h} \mathbf{I}
$$

The function $f^{*}$, introduced in Tvergaard and Needleman (1984), is given by

$$
f^{*}=\left\{\begin{array}{cc}
f, & f<f_{c} \\
f_{c}+\left(1 / q_{1}-f_{c}\right)\left(f-f_{c}\right) /\left(f_{f}-f_{c}\right), & f \geq f_{c}
\end{array}\right.
$$




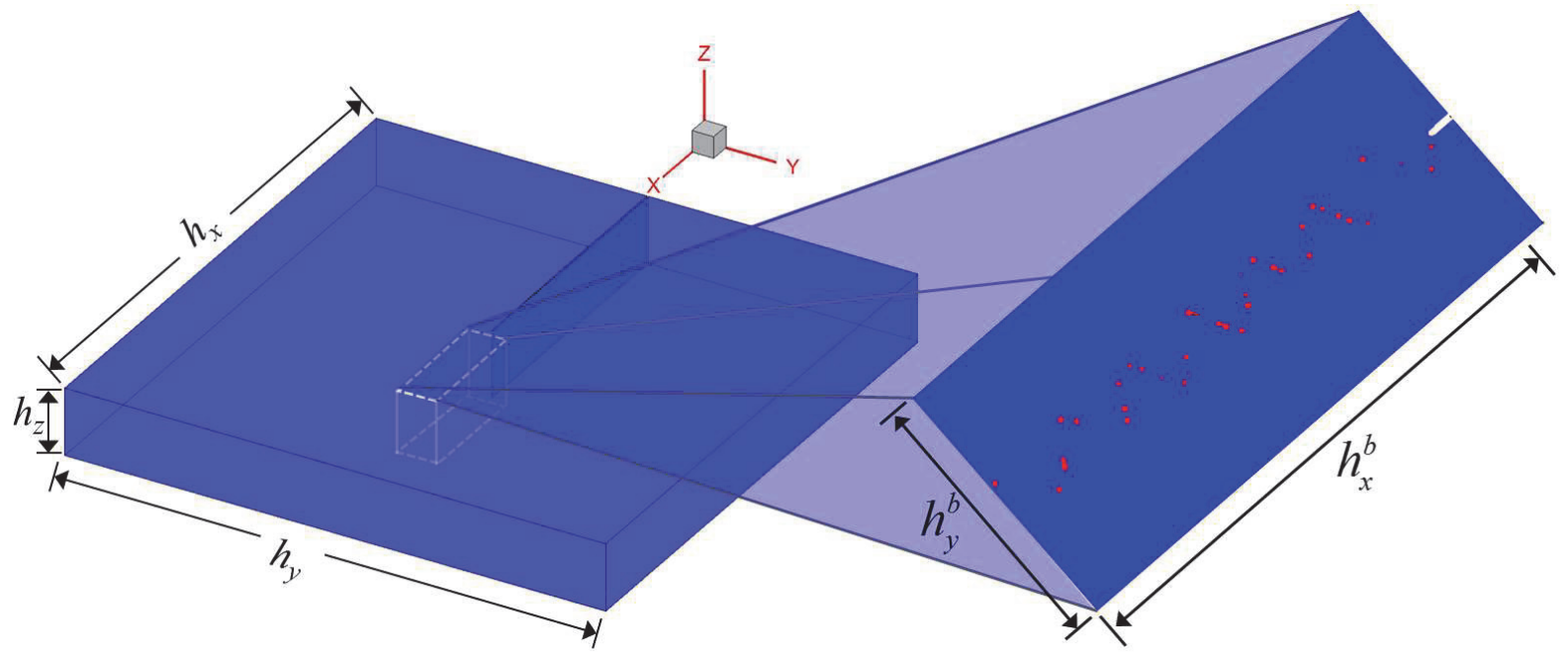

Figure 1: Sketch of the initially cracked block analyzed with a magnified view of the sinusoidal distribution of large particles on the top plane. For the distribution shown, amplitude is $10 e_{x}$, wavelength is $30 e_{x}$ and mean particle spacing is $4 e_{x}$, where $e_{x}$ is a normalization length parameter.

where the values $f_{c}=0.12$ and $f_{f}=0.25$ are used. The rate of deformation tensor written as the sum of an elastic part, $\mathbf{d}^{e}=\mathbf{L}^{-1}: \hat{\boldsymbol{\sigma}}$, a viscoplastic part, $\mathbf{d}^{p}$, and a part due to thermal straining, $\mathbf{d}^{\Theta}=\alpha \dot{\Theta} \mathbf{I}$, so that

$$
\mathbf{d}=\mathbf{L}^{-1}: \hat{\boldsymbol{\sigma}}+\alpha \dot{\Theta} \mathbf{I}+\mathbf{d}^{p}
$$

Here, $\hat{\boldsymbol{\sigma}}$ is the Jaumann rate of Cauchy stress, $\Theta$ is the temperature, $\alpha=1 \times 10^{-5} / \mathrm{K}$ is the thermal expansion coefficient and $\mathbf{L}$ is the tensor of isotropic elastic moduli. The plastic part of the strain rate, $\mathbf{d}^{p}$, is given by Pan et al. (1983)

$$
\mathbf{d}^{p}=\left[\frac{(1-f) \overline{\bar{\epsilon}}}{\boldsymbol{\sigma}: \frac{\partial \phi}{\partial \boldsymbol{\sigma}}}\right] \frac{\partial \phi}{\partial \boldsymbol{\sigma}}
$$

The matrix plastic strain rate, $\dot{\bar{\epsilon}}$, is given by

$$
\dot{\bar{\epsilon}}=\dot{\epsilon}_{0}\left[\frac{\bar{\sigma}}{g(\bar{\epsilon}, \Theta)}\right]^{1 / m} \quad, \quad g(\bar{\epsilon}, \Theta)=\sigma_{0} G(\Theta)\left[1+\bar{\epsilon} / \epsilon_{0}\right]^{N}
$$

with $\bar{\epsilon}=\int \dot{\bar{\epsilon}} d t$ and $\epsilon_{0}=\sigma_{0} / E$. In the calculations here $E=70 \mathrm{GPa}, \nu=0.3, \sigma_{0}=300 \mathrm{MPa}$ $\left(\epsilon_{0}=\sigma_{0} / E=0.00429\right), N=0.1, m=0.01$ and $\dot{\epsilon}_{0}=10^{3} \mathrm{~s}^{-1}$. Adiabatic conditions are assumed so that

$$
\rho c_{p} \frac{\partial \Theta}{\partial t}=\chi \boldsymbol{\tau}: \mathbf{d}^{p}
$$

with $\rho=7600 \mathrm{~kg} / \mathrm{m}^{3}=7.6 \times 10^{-3} \mathrm{MPa} /(\mathrm{m} / \mathrm{s})^{2}, c_{p}=465 \mathrm{~J} /\left(\mathrm{kg}{ }^{\circ} \mathrm{K}\right), \chi=0.9$, and the temperature-dependence of the flow strength is given by

$$
G(\Theta)=1+b_{G} \exp \left(-c\left[\Theta_{0}-273\right]\right)\left[\exp \left(-c\left[\Theta-\Theta_{0}\right]\right)-1\right]
$$


with $b_{G}=0.1406$ and $c=0.00793 / \mathrm{K}$. In Eq. (8), $\Theta$ and $\Theta_{0}$ are in $\mathrm{K}$ and $\Theta_{0}=293 \mathrm{~K}$. Also, the initial temperature is taken to be uniform and $293 \mathrm{~K}$. The initial void volume fraction is taken to be zero and the evolution of the void volume fraction is governed by

$$
\dot{f}=(1-f) \mathbf{d}^{p}: \mathbf{I}+\dot{f}_{\text {nucl }}
$$

where the first term on the right hand side of Eq. (9) accounts for void growth and the second term accounts for void nucleation.

Eight point Gaussian integration is used in each twenty-node element for integrating the internal force contributions and twenty-seven point Gaussian integration is used for the element mass matrix. Lumped masses are used so that the mass matrix is diagonal. The discretized equations are integrated using the explicit Newmark $\beta$-method $(\beta=0)$ (Belytschko et al., 1976). The constitutive updating is based on the rate tangent modulus method in Peirce et al. (1984), while material failure is implemented via the element vanish technique in Tvergaard (1982b). When the value of the void volume fraction $f$ at an integration point reaches $0.9 f_{f}$, the value of $f$ is kept fixed so that the material deforms with a very small flow strength. The entire element is taken to vanish when three of the eight integration points in the element have reached this stage.

\section{Microstructure generation}

In the calculations the material microstructure is characterized by two populations of void nucleating second phase particles: (i) uniformly distributed small particles that are modeled by plastic strain controlled void nucleation; and (ii) large particles that are modeled as stress controlled nucleation sites. The large particles introduce microstructural length scale into the formulation as shown in Srivastava et al. (2014); Osovski et al. (2015a). In each case, void nucleation is assumed to be described by a normal distribution, Chu and Needleman (1980).

For plastic strain nucleation

$$
\dot{f}_{n u c l}=k_{\epsilon} \dot{\bar{\epsilon}}, k_{\epsilon}=\frac{f_{N}}{s_{N} \sqrt{2 \pi}} \exp \left[-\frac{1}{2}\left(\frac{\bar{\epsilon}-\epsilon_{N}}{s_{N}}\right)^{2}\right]
$$

with $f_{N}=0.04, \epsilon_{N}=0.3$ and $s_{N}=0.1$.

For stress controlled nucleation

$$
\dot{f}_{\text {nucl }}=k_{\sigma}\left[\dot{\bar{\sigma}}+\dot{\sigma}_{h}\right], k_{\sigma}=\frac{f_{N}}{s_{N} \sqrt{2 \pi}} \exp \left[-\frac{1}{2}\left(\frac{\bar{\sigma}+\sigma_{h}-\sigma_{N}}{s_{N}}\right)^{2}\right]
$$

if $\left(\bar{\sigma}+\sigma_{h}\right)$ is at its maximum over the deformation history. Otherwise $k_{\sigma}=0$. The value of $f_{N}$ in Eq. (11) at a point $\left(x_{0}, y_{0}, z_{0}\right)$ in the initial undeformed configuration, for a particle of radius $r_{c}$ centered at $\left(x_{c}, y_{c}, z_{c}\right)$ is

$$
f_{N}=\left\{\begin{array}{cc}
\bar{f}_{N} & \text { for } \sqrt{\left(x_{0}-x_{c}\right)^{2}+\left(y_{0}-y_{c}\right)^{2}+\left(z_{0}-z_{c}\right)^{2}} \leq r_{c} \\
0 & \text { for } \sqrt{\left(x_{0}-x_{c}\right)^{2}+\left(y_{0}-y_{c}\right)^{2}+\left(z_{0}-z_{c}\right)^{2}}>r_{c}
\end{array}\right.
$$


The values $\bar{f}_{N}=0.04, \sigma_{N} / \sigma_{0}=1.5$ and $s_{N} / \sigma_{0}=0.2$ are used in the calculations. The particle radius $r_{c}=1.5 e_{x}$ (where $e_{x}$ is the in-plane element dimension) is kept fixed. The controlled microstructure is characterized by the sinusoidal distribution of particles (modeled as stress controlled nucleation sites) with a given mean particle spacing, $l_{0}$, in the region, $h_{x}^{b} \times h_{y}^{b} \times h_{z}$, Fig. 1, in front of the initial crack tip. In terms of $e_{x}, h_{x}^{b}=200 e_{x}, h_{y}^{b}=60 e_{x}$, and $h_{z}=50 e_{x}$. In order to generate the controlled microstructure with sinusoidal distribution of particles for a given amplitude, $A$, and wavelength, $\lambda$, of the sinusoidal profile, and mean particle spacing, $l_{0}$, the number of particles, $N_{i n c l}$, in the region $h_{x}^{b} \times h_{y}^{b} \times h_{z}$ in front of the initial crack tip is varied. For a given mean particle spacing and sinusoidal profile,

$$
N_{\text {incl }}=\frac{A_{\text {surf }}}{\left(l_{0}\right)^{2}}, A_{\text {surf }}=h_{z} \times \int_{0}^{h_{x}^{b}} \sqrt{1+\frac{2 \pi}{\lambda} A \cos ^{2}\left(\frac{2 \pi}{\lambda} x\right)} d x
$$

The $x_{c}$ and $z_{c}$ coordinates of $N_{\text {incl }}$ number of particle centers are then determined using a random number generator with $y_{c}=A \sin \left(2 \pi x_{c} / \lambda\right)$ and an imposed restriction that the center to center distance of two neighboring particles is at least twice the particle radius, $r_{c}$. Hence the particles are constrained to lie along a sinusoidal profile but within the profile the particle distribution is random with a specified mean particle spacing. A controlled microstructure characterized by sinusoidal distribution of particles with $A=10 e_{x}$ and $\lambda=$ $30 e_{x}$ for $l_{0}=4 e_{x}$ generated using this procedure is shown in Fig. 1. Also for the purpose of comparison, microstructures with random distribution of particles are also generated. The random distribution of particles for a given mean particle spacing are generated by varying the number of particles, $N_{\text {incl }}$, in front of the initial crack tip. For a given mean particle spacing for random distribution, $N_{\text {incl }}=V_{u} /\left(l_{0}\right)^{3}$ where $V_{u}=h_{x}^{b} \times h_{y}^{b} \times h_{z}$. For the random distribution all three coordinates of the particle centers within $V_{u}$ are determined using a random number generator with the restriction that the center to center distance of two neighboring particles is at least twice the particle radius.

\section{Numerical results}

A monotonically increasing remote stress intensity factor, $K_{I}(t)$, with $\dot{K}_{I} /\left(\dot{\epsilon}_{0} \sigma_{0} \sqrt{e_{x}}\right)=$ 1359.4 as in Srivastava et al. (2014) is prescribed and calculations continue until the projected crack length, $\Delta a$, in the propagation direction (along $x$-axis) approaches $h_{x}^{b}$, Fig. 1 . The calculations are carried out for two mean particle spacings, $l_{0}=4 e_{x}$ and $8 e_{x}$. These two values of $l_{0}$ were chosen because in Srivastava et al. (2014) three dimensional random distribution of particles with $l_{0}=4 e_{x}$ led to multiple void interaction (Tvergaard and Hutchinson, 2002) dominated crack growth whereas random distribution of particles with $l_{0}=8 e_{x}$ resulted in void by void dominated (Tvergaard and Hutchinson, 2002) mode of crack growth. The transition from void by void to multiple void interaction dominated mode of crack growth can be sensitive to material parameters and applied loading rate, however, the material parameters and loading rate considered here are same as in Srivastava et al. (2014), so permitting a direct comparison. For each mean particle spacing controlled sinusoidal distribution of particles are generated for a range of amplitude, $A$, and wavelength, $\lambda$, of 


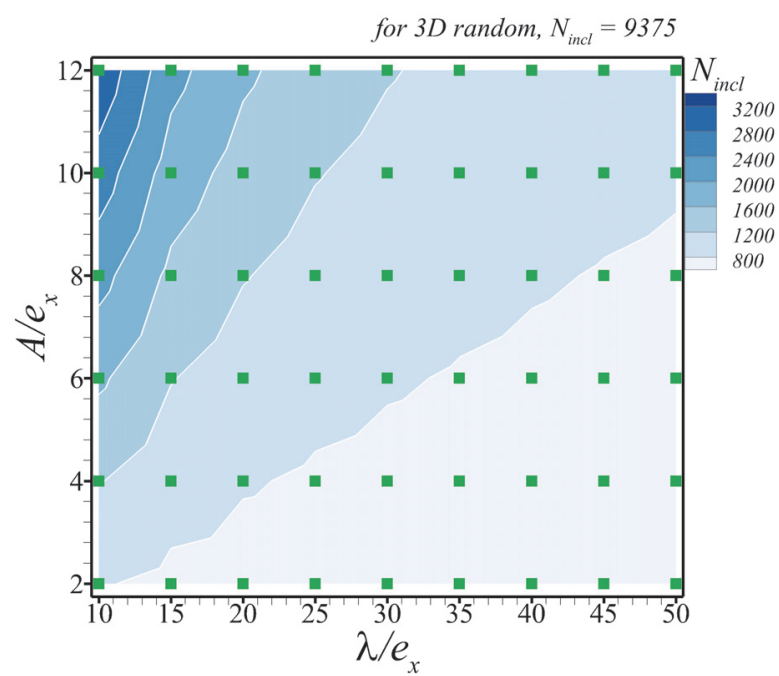

(a)

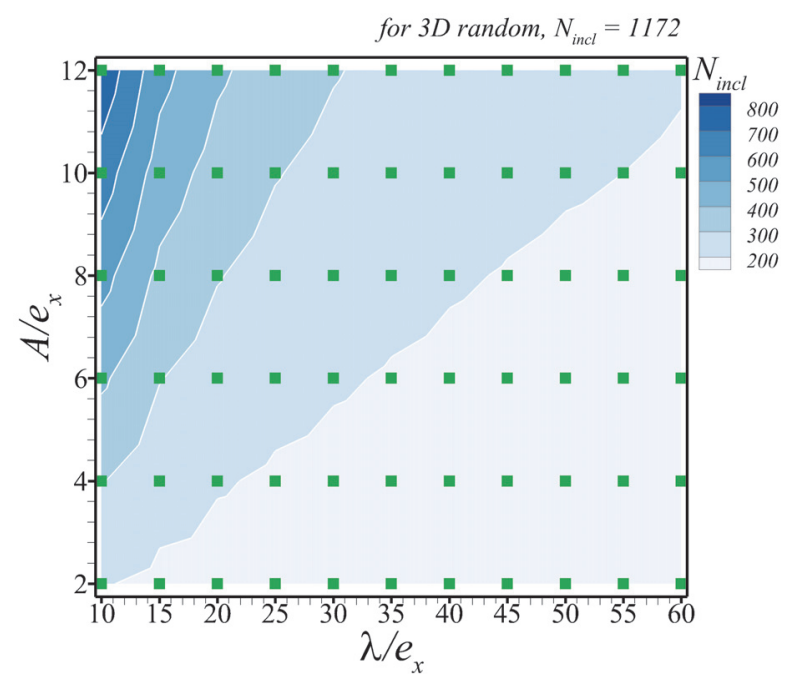

(b)

Figure 2: Variation of number of particles, $N_{\text {incl }}$, with amplitude, $A$, and wavelength, $\lambda$, of the sinusoidal distribution of the particles with mean particle spacing (a) $l_{0}=4 e_{x}$ and (b) $l_{0}=8 e_{x}$. The filled squares represent the combination of $A$ and $\lambda$ analyzed for each $l_{0}$.

the sinusoidal profile. The variation in the number of particles, $N_{i n c l}$, with the variation in $A$ and $\lambda$ of the sinusoidal distribution are shown in Fig. 2. In Fig. 2, filled squares represent the combination of $A$ and $\lambda$ considered for each mean particle spacing. For $l_{0}=4 e_{x}$ in total 54 cases are analyzed whereas for $l_{0}=8 e_{x}$ in total 66 cases are analyzed. For both the mean particle spacings, $l_{0}=4 e_{x}$ and $8 e_{x}$, three dimensional random distribution of particles are also considered, and for a fixed $l_{0}$, calculations are carried out for five realizations of the particle centers.

Crack growth resistance curves, plots of $J$ versus $\Delta a$, are calculated and, as in Srivastava et al. (2014), the crack growth resistance is characterized by two parameters, the fracture toughness, $J_{I C}$, and the tearing modulus, $T_{R}$. The value of $J$ is calculated from the applied value of $K_{I}$ using the relation, Rice (1968)

$$
J=K_{I}^{2} \frac{\left(1-\nu^{2}\right)}{E}
$$

and, as in Srivastava et al. (2014), $\Delta a$ is obtained by averaging the maximum projected length of contours of $f=0.1$ taken over 10 equally spaced planes through the thickness. As noted in Osovski et al. (2015a), this procedure of estimating $\Delta a$ does not ensure that the calculated values of $\Delta a$ always represents a continuous crack. In most cases, the micro-cracks that nucleate ahead of the dominant crack rather quickly link up with the dominant crack and hence $\Delta a$ is, at least approximately, a measure of the length of the dominant crack. The values of $J_{I C}$ are obtained using a procedure mimicking the ASTM E1820-11 (2011) standard, where a power law of the form $J /\left(\sigma_{0} e_{x}\right)=C_{1}\left(\Delta a / e_{x}\right)^{C_{2}}$ is fit to the portion of the $J-R$ curve 
in between two exclusion lines, $J / e_{x}=2 \sigma_{0}\left(\Delta a / e_{x}-1.5\right)$ and $J / e_{x}=2 \sigma_{0}\left(\Delta a / e_{x}-15\right)$. The normalized fracture toughness value, $J /\left(\sigma_{0} e_{x}\right)$, is then defined as the intersection of the curve $J /\left(\sigma_{0} e_{x}\right)=C_{1}\left(\Delta a / e_{x}\right)^{C_{2}}$ and the line $J / e_{x}=2 \sigma_{0}\left(\Delta a / e_{x}-2\right)$.

The non-dimensional tearing modulus, Paris et al. (1979), is given by

$$
T_{R}=\left(\frac{E}{\sigma_{0}^{2}}\right) \frac{d J}{d(\Delta a)}
$$

Here, $T_{R}$ is calculated using the slope of a linear fit to the $J-R$ curve in the range $50 \leq$ $\Delta a / e_{x} \leq 150$

\subsection{Crack growth resistance}

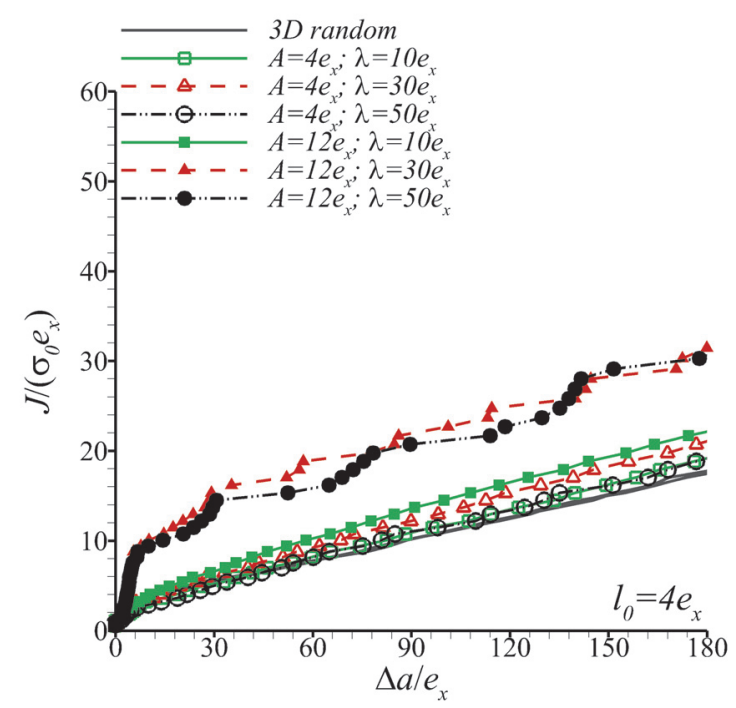

(a)

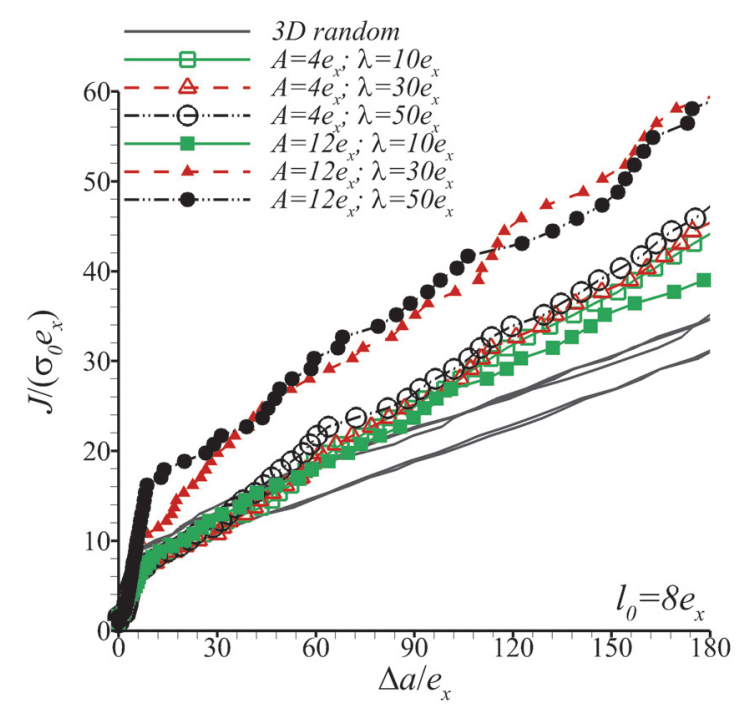

(b)

Figure 3: Applied normalized $J, J /\left(\sigma_{0} e_{x}\right)$, versus normalized crack extension, $\Delta a / e_{x}$, curves for two values of amplitude, $A$, and for each $A$ three values of wavelength, $\lambda$, of the sinusoidal distribution of the particles together with five realizations of the 3D random distribution of the particles with mean particle spacing (a) $l_{0}=4 e_{x}$ and (b) $l_{0}=8 e_{x}$.

Fig. 3 shows crack growth resistance curves, $J-R$ curves, for two values of amplitude, $A=4 e_{x}$ and $12 e_{x}$, and for each value of $A$ curves are shown for three values of wavelength, $\lambda=10 e_{x}, 30 e_{x}$ and $50 e_{x}$, of the sinusoidal distribution of particles. For comparison purposes, curves for five realizations of the 3D random distribution of the particles with mean particle spacings, $l_{0}=4 e_{x}$ and $8 e_{x}$ are also shown. As seen in Fig. 3 the level of the $J-R$ curves (i.e. the value of $J$ at a given value of $\Delta a$ ) is smaller for $l_{0}=4 e_{x}$ than $l_{0}=8 e_{x}$ for both sinusoidal and random distributions of particles. For the sinusoidal distribution of particles with a fixed $l_{0}$, the crack growth resistance simultaneously depends on both amplitude and wavelength of the profile. For relatively small values of the wavelength $\lambda$ of the sinusoidal 
profile the effect of the amplitude $A$ on the crack growth resistance is small. Similarly, for relatively small values of $A$ the effect of $\lambda$ on the crack growth resistance is small. In general, for a fixed particle spacing $l_{0}$, an increase in crack growth resistance occurs when both the amplitude and the wavelength are increased. An increase in both the amplitude and the wavelength of the sinusoidal profile with a fixed $l_{0}$ implies an increased number of particles along the sinusoidal profile, Fig. 2. For a sufficiently large wavelength and a sufficiently large amplitude of the sine, the crack growth resistance for the sinusoidal distribution is greater than that for the random distributions having the same $l_{0}$.
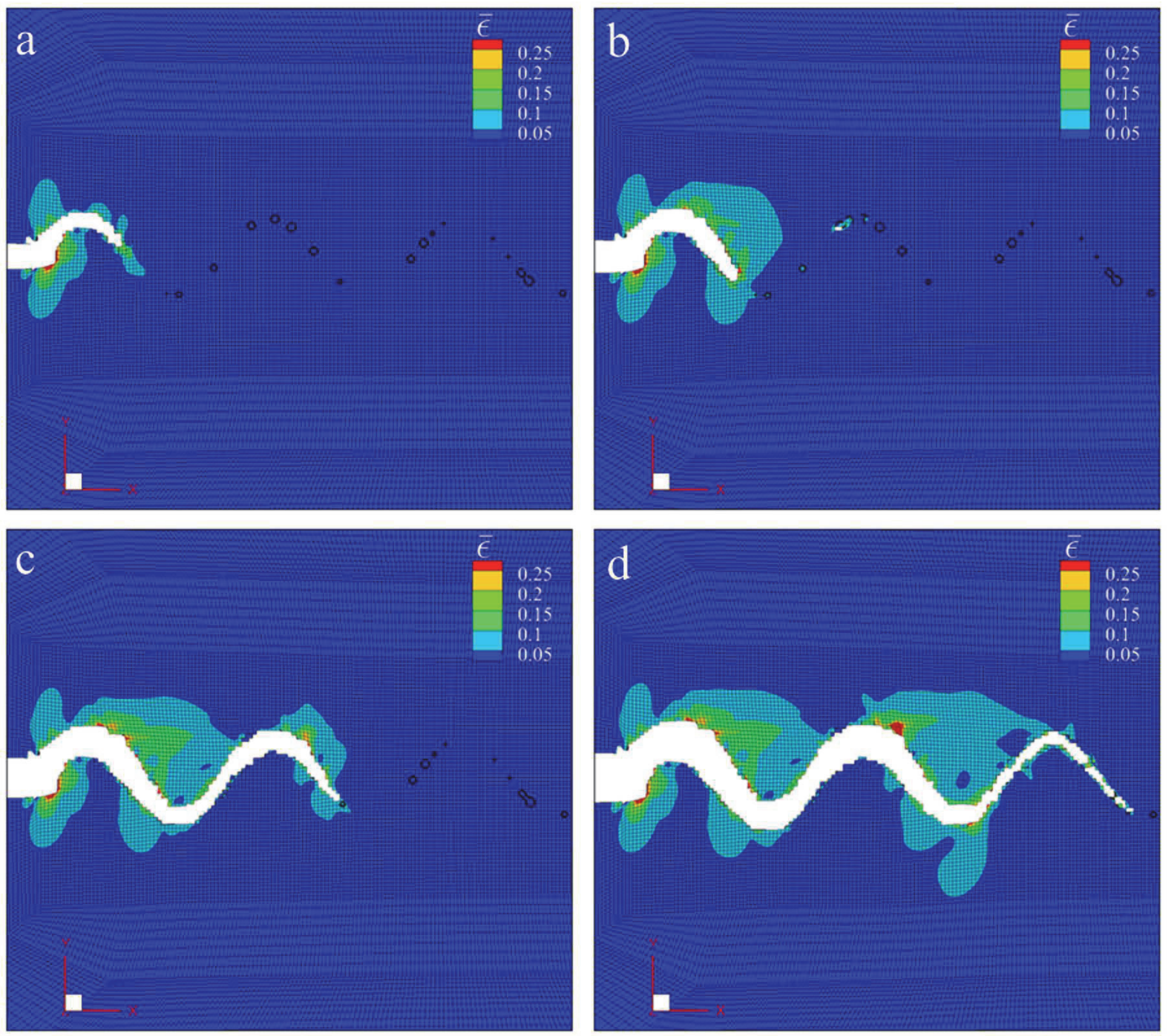

Figure 4: Crack path and distribution of equivalent plastic strain, $\bar{\epsilon}$, for a sinusoidal distribution of particles with amplitude, $A=10 e_{x}$, and wavelength, $\lambda=50 e_{x}$, for mean particle spacing, $l_{0}=4 e_{x}$, at applied normalized $J$, (a) $J /\left(\sigma_{0} e_{x}\right)=10.78$, (b) $J /\left(\sigma_{0} e_{x}\right)=14.67$, (c) $J /\left(\sigma_{0} e_{x}\right)=19.16$, and (d) $J /\left(\sigma_{0} e_{x}\right)=24.25$. The open circles represent the location of particles on the $z$-plane shown. 

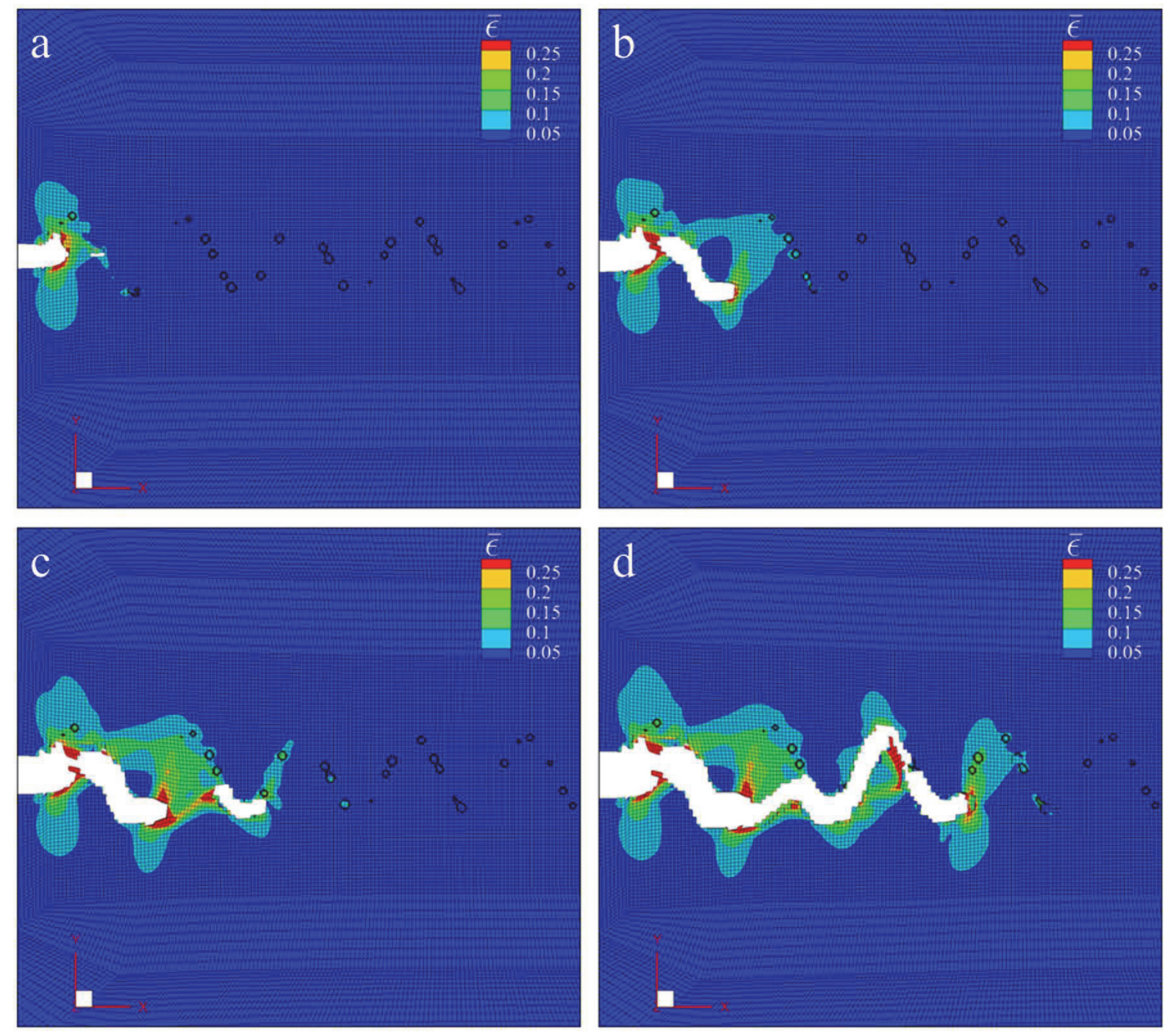

Figure 5: Crack path and distribution of equivalent plastic strain, $\bar{\epsilon}$, for a sinusoidal distribution of particles with amplitude, $A=10 e_{x}$, and wavelength, $\lambda=30 e_{x}$, for mean particle spacing, $l_{0}=4 e_{x}$, at applied normalized $J$, (a) $J /\left(\sigma_{0} e_{x}\right)=10.78$, (b) $J /\left(\sigma_{0} e_{x}\right)=14.67$, (c) $J /\left(\sigma_{0} e_{x}\right)=19.16$, and (d) $J /\left(\sigma_{0} e_{x}\right)=24.25$. The open circles represent the location of particles on the $z$-plane shown.

The $J-R$ curves for sinusoidal distributions of particles with sufficiently large amplitudes $A$ and sufficiently large wavelengths $\lambda$ show near jump increases in the value of $J$ and/or $\Delta a$, as seen in Fig. 3 for both $l_{0}=4 e_{x}$ and $8 e_{x}$. It is possible that not all jumps were captured in the plotted $J-R$ curves since jumps may have occurred between plotted points. The origin of such jumps in the $J-R$ curves, is revealed by the evolution of the crack paths and of the distributions of matrix effective plastic strain, $\bar{\epsilon}$, in Figs. 4 to 6.

Fig. 4 shows the crack path and distribution of matrix plastic strain $\bar{\epsilon}$ for a sinusoidal distribution of particles with amplitude $A=10 e_{x}$ and wavelength $\lambda=50 e_{x}$ for $l_{0}=4 e_{x}$ 

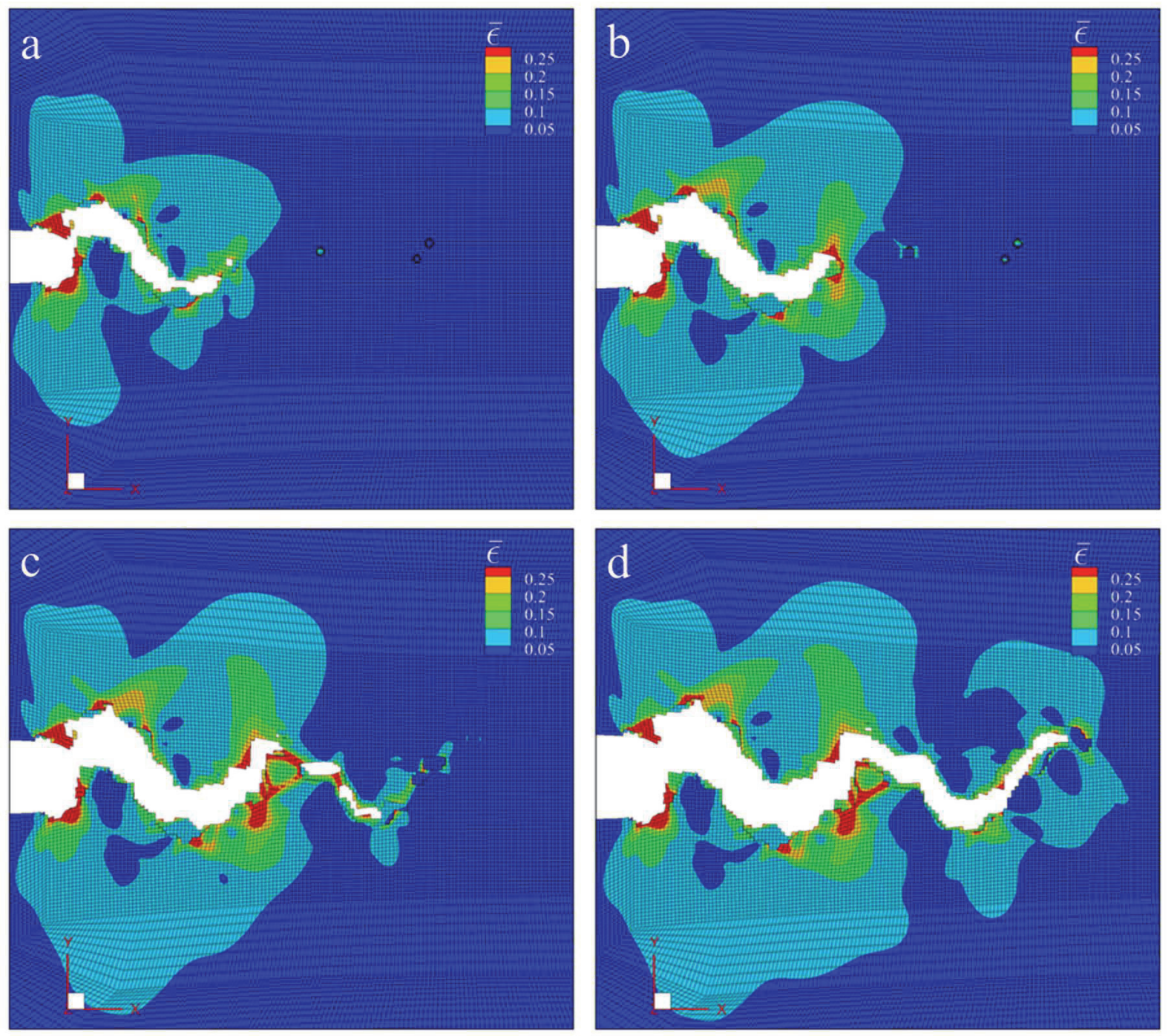

Figure 6: Crack path and distribution of equivalent plastic strain, $\bar{\epsilon}$, for a sinusoidal distribution of particles with amplitude, $A=10 e_{x}$, and wavelength, $\lambda=50 e_{x}$, for mean particle spacing, $l_{0}=8 e_{x}$, at applied normalized $J,(\mathrm{a}) J /\left(\sigma_{0} e_{x}\right)=29.24$, (b) $J /\left(\sigma_{0} e_{x}\right)=36.23$, (c) $J /\left(\sigma_{0} e_{x}\right)=43.11$, and (d) $J /\left(\sigma_{0} e_{x}\right)=50.60$. The open circles represent the location of particles on the $z$-plane shown.

at four values of applied $J$. This is a case where the crack follows the sinusoidal particle distribution, as seen in Fig. 4. In this case, after the growing crack reaches a crest or trough of the sinusoidal profile, further crack advance involves a large deflection of the crack path, Fig. 4b. This results in much of the crack growth occurring in a direction orthogonal to the initial crack plane and thus not contributing to increasing $\Delta a$. On the other hand, the plastic dissipation between a crest and trough (or between a trough and crest) contributes to the value of $J$. This can result in a near jump increase in the value of $J$. A decrease in the amplitude of the sinusoidal profile decreases the extent of crack deflection and hence 


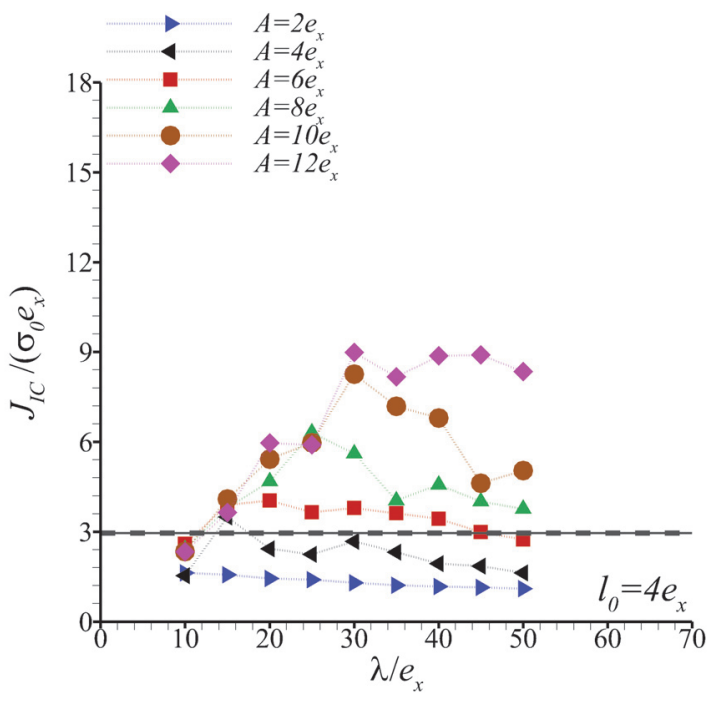

(a)

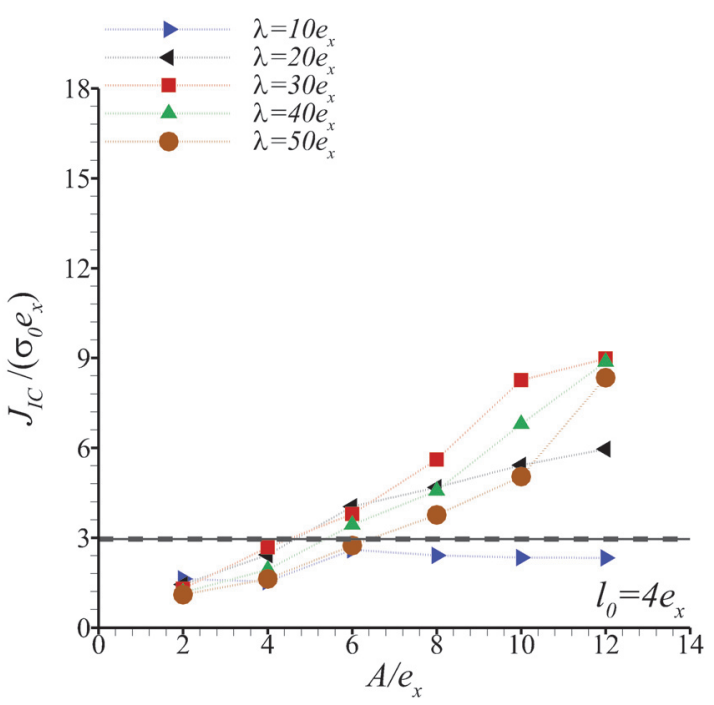

(c)

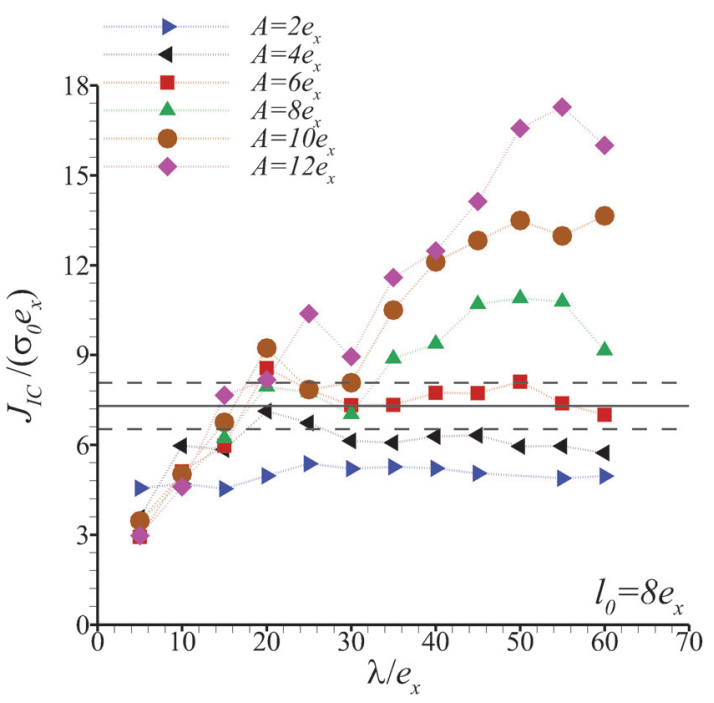

(b)

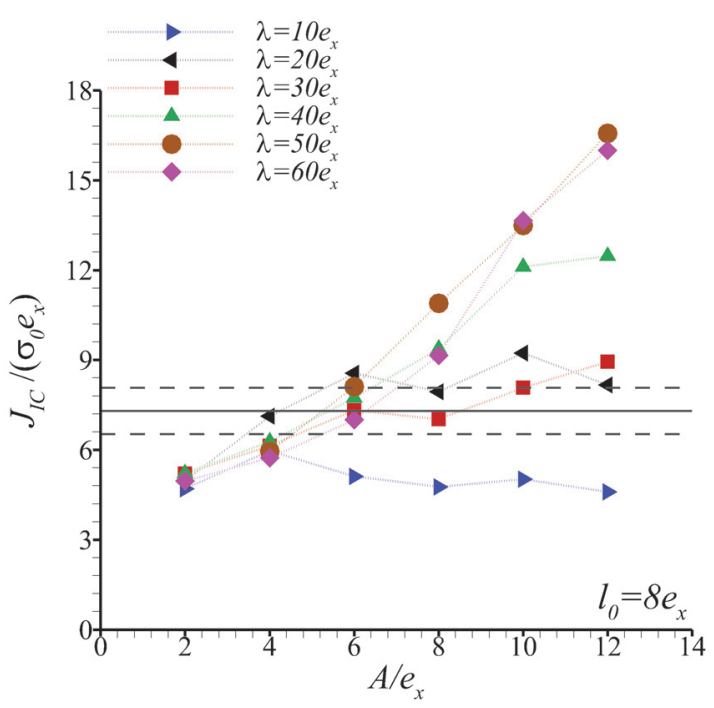

(d)

Figure 7: Variation of normalized fracture toughness, $J_{I C} /\left(\sigma_{0} e_{x}\right)$, with normalized wavelength, $\lambda / e_{x}$, of the sinusoidal distribution of the particles for fixed values of amplitude, $A$, for mean particle spacing (a) $l_{0}=4 e_{x}$ and $(\mathrm{b}) l_{0}=8 e_{x}$; and variation of $J_{I C} /\left(\sigma_{0} e_{x}\right)$, with normalized amplitude, $A / e_{x}$, of the sinusoidal distribution of the particles for fixed values of $\lambda$ for (c) $l_{0}=4 e_{x}$ and (d) $l_{0}=8 e_{x}$. The horizontal solid lines mark the average $J_{I C} /\left(\sigma_{0} e_{x}\right)$ values for five realizations of 3D random distribution of particles with the same $l_{0}$ values while the horizontal dashed lines mark the standard errors for realizations.

tends to decrease the extent of the jumps in the $J-R$ curve.

Fig. 5 shows the crack path and distributions of matrix effective plastic strain, $\bar{\epsilon}$, at four levels of applied $J$ for a sinusoidal distribution of particles with the same amplitude, $A=10 e_{x}$, and the same mean particle spacing, $l_{0}=4 e_{x}$, as in Fig. 4 , but with the wavelength 


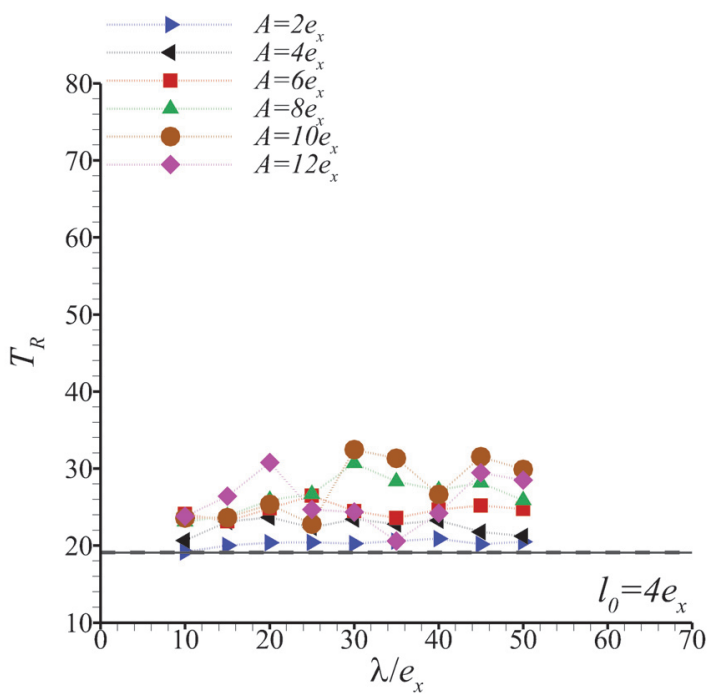

(a)

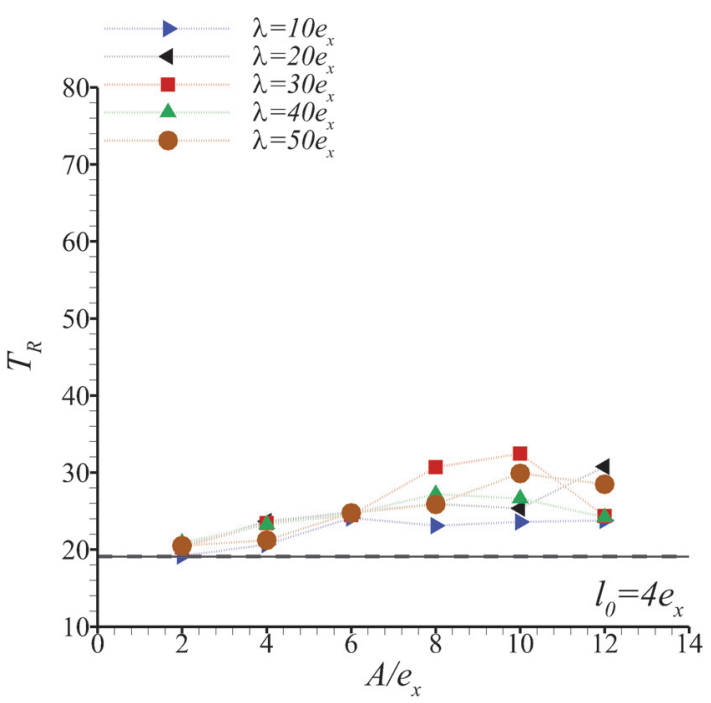

(c)

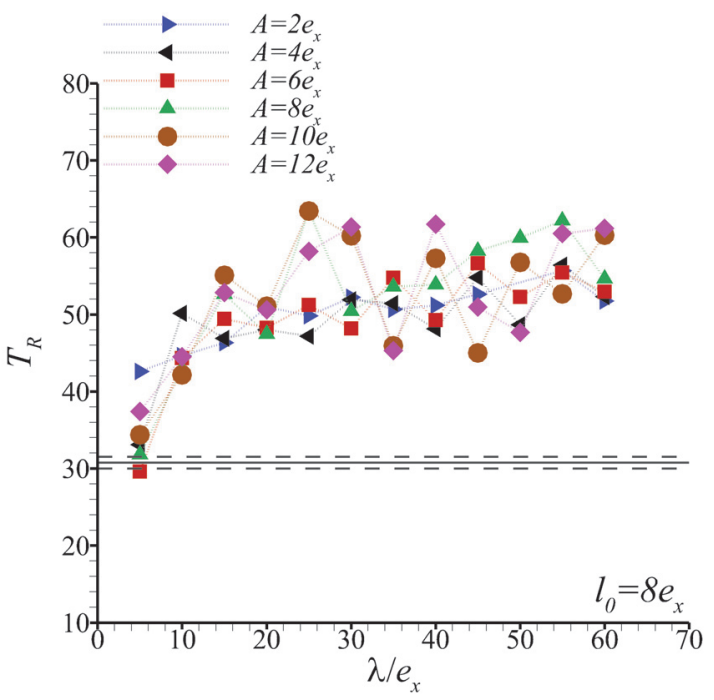

(b)

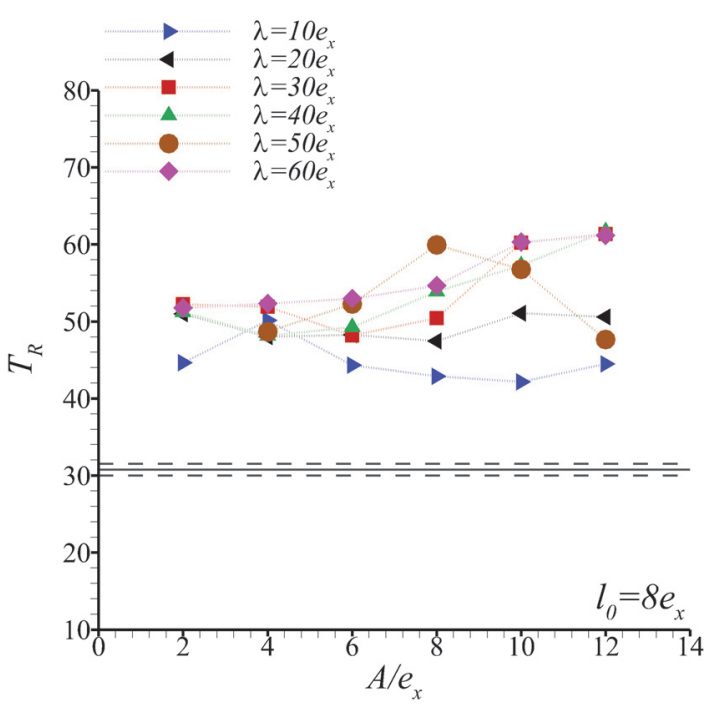

(d)

Figure 8: Variation of tearing modulus, $T_{R}$, with normalized wavelength, $\lambda / e_{x}$, of the sinusoidal distribution of the particles for fixed values of amplitude, $A$, for mean particle spacing (a) $l_{0}=4 e_{x}$ and (b) $l_{0}=8 e_{x}$; and variation of $T_{R}$ with normalized amplitude, $A / e_{x}$, of the sinusoidal distribution of the particles for fixed values of $\lambda$ for (c) $l_{0}=4 e_{x}$ and (d) $l_{0}=8 e_{x}$. The horizontal solid lines mark the average $T_{R}$ values for five realizations of $3 \mathrm{D}$ random distribution of particles with the same $l_{0}$ values while the horizontal dashed lines mark the standard errors for realizations.

decreased to $\lambda=30 e_{x}$. In this case the crack path does not follow the sinusoidal particle distribution. As seen in Figs. 5a and c, a micro-crack nucleates ahead of the dominant crack which is then followed by localization of deformation between the main crack and the nucleated micro-crack. Crack growth subsequently occurs by coalescence of the main crack 
and the micro-crack. This may also lead to jumps in the $J-R$ curve if the micro-crack is sufficiently far away from the main crack. A decrease in the wavelength of the sinusoidal particle profile necessarily decreases the distance between the main crack and any nucleated micro-cracks. Hence, this decreases the amplitude of the jumps in the $J-R$ curve.

Fig. 6 shows the evolution of the crack path and the distribution of $\bar{\epsilon}$, for a sinusoidal particle distribution with a mean particle spacing $l_{0}=8 e_{x}$, an amplitude $A=10 e_{x}$ and a wavelength $\lambda=50 e_{x}$ (the same values of amplitude and wavelength as in Fig. 4 where $\left.l_{0}=4 e_{x}\right)$. As seen in Fig. 6, the crack tends to follow the sinusoidal particle distribution. The crack growth resistance (the level of the $J-R$ curve) is increased from the corresponding case with a mean particle spacing of $l_{0}=4 e_{x}$, the case in Fig. 4 , since the larger particle spacing results in increased plastic dissipation per unit crack advance due to the reduced number of particles along the sinusoidal profile. Crack growth in Fig. 6 generally involves nucleation of micro-voids and/or micro-cracks ahead of the dominant crack which is followed by localization of deformation between the main crack and the nucleated micro-voids and/or micro-cracks. The main crack subsequently coalesces with the nucleated micro-voids/microcracks.

The variation of $J_{I C} /\left(\sigma_{0} e_{x}\right)$ with normalized wavelength, $\lambda / e_{x}$, for the sinusoidal distribution of particles with mean particle spacings $l_{0}=4 e_{x}$ and $8 e_{x}$ is shown in Figs. $7 \mathrm{a}$ and 7b. As seen in Figs. 7a and b, the value of $J_{I C}$ increases with increasing $\lambda$ up to a certain value of $\lambda$ and then tends to a plateau. For a specified value of mean particle spacing, $l_{0}$, this value of $\lambda$ depends on the distribution amplitude, $A$. It decreases with decreasing $A$ and increases with increasing $A$. For $A<6 e_{x}$, for both $l_{0}=4 e_{x}$ and $8 e_{x}, J_{I C}$ is nearly independent of $\lambda$ for the range of wavelengths considered.

The variation of $J_{I C} /\left(\sigma_{0} e_{x}\right)$, with normalized amplitude, $A / e_{x}$, for the sinusoidal distribution of the particles with mean spacings $l_{0}=4 e_{x}$ and $8 e_{x}$ is shown in Figs. 7c and 7d. From Figs. $7 \mathrm{c}$ and $7 \mathrm{~d}$, it can be seen that the value of $J_{I C}$ increases with increasing $A$ up to a certain value of $A$ and then tends to a plateau. For a fixed value of $l_{0}$, this critical value of $A$ depends on $\lambda$ and it decreases with decrease in $\lambda$, and vice versa. For $\lambda=10 e_{x}$ for both $l_{0}=4 e_{x}$ and $8 e_{x}, J_{I C}$ is nearly independent of $A$ for the range of amplitudes considered. Also the maximum value of $J_{I C}$ for sinusoidal distributions (for the range of $A$ and $\lambda$ considered here) of particles, with $l_{0}=4 e_{x}$ is approximately 3 times that for the random distributions having the same value of $l_{0}$. With a mean particle spacing $l_{0}=8 e_{x}$, the maximum value of $J_{I C}$ is about 2.4 times that of the corresponding random distribution.

The variation of $T_{R}$ with normalized wavelength, $\lambda / e_{x}$, of the sinusoidal distributions of particles with $l_{0}=4 e_{x}$ and $8 e_{x}$ are shown in Figs. $8 \mathrm{a}$ and $8 \mathrm{~b}$. The variation of $T_{R}$, with normalized amplitude, $A / e_{x}$, of the sinusoidal distribution of the particles having mean particle spacings of $l_{0}=4 e_{x}$ and $8 e_{x}$ are shown in Figs. $8 \mathrm{c}$ and $8 \mathrm{~d}$. For $l_{0}=4 e_{x}$, the value of $T_{R}$ is nearly independent of wavelength $\lambda$ but slightly increases with increasing amplitude $A$ of the particle distribution. On average, the value of $T_{R}$ for the sinusoidal distributions is only slightly greater than the value of $T_{R}$ for the corresponding random distribution. For $l_{0}=8 e_{x}$, the value of $T_{R}$ increases slightly with increasing $\lambda$ but is nearly independent of amplitude $A$. Generally, for the sinusoidal distributions of particles with mean spacing $l_{0}=8 e_{x}$ and wavelength $\lambda>20 e_{x}$, the value of $T_{R}$ is about 1.5 to 2 times that of the 
corresponding random distribution.

For all cases analyzed, contour plots, that give a visual overview, of the variation of $J_{I C} /\left(\sigma_{0} e_{x}\right)$ and $T_{R}$ with normalized amplitude, $A / e_{x}$, and normalized wavelength, $\lambda / e_{x}$ are shown in the supplementary material. The greatest increase of $J_{I C} /\left(\sigma_{0} e_{x}\right)$ occurs when amplitude, $A$, and wavelength, $\lambda / 4$, are both increased while keeping the ratio of the amplitude to quarter wavelength, $A /(\lambda / 4) \approx 1$. The value of $T_{R}$ also tends to increase when $A$ and $\lambda / 4$ increase together, but there is considerable variation about $A \approx \lambda / 4$, especially for $l_{0}=8 e_{x}$.

\subsection{Fracture surface roughness}

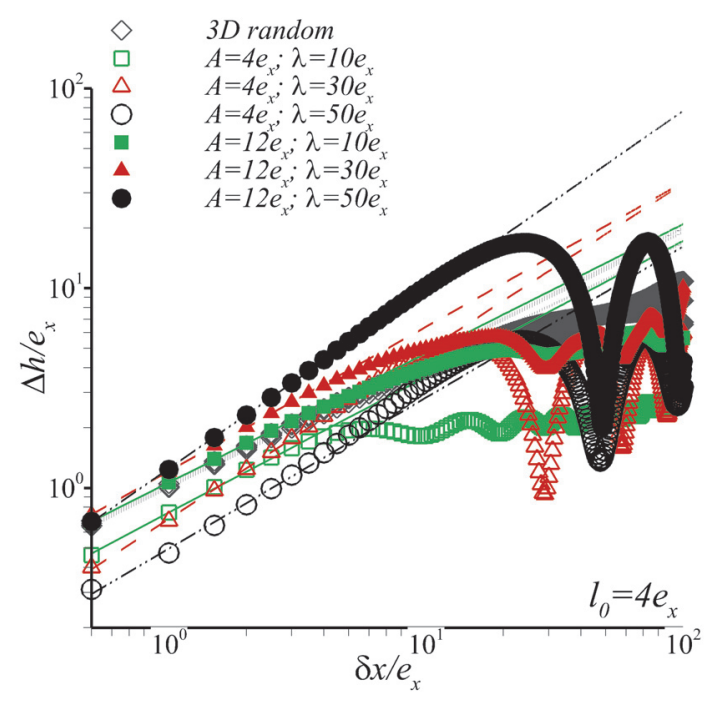

(a)

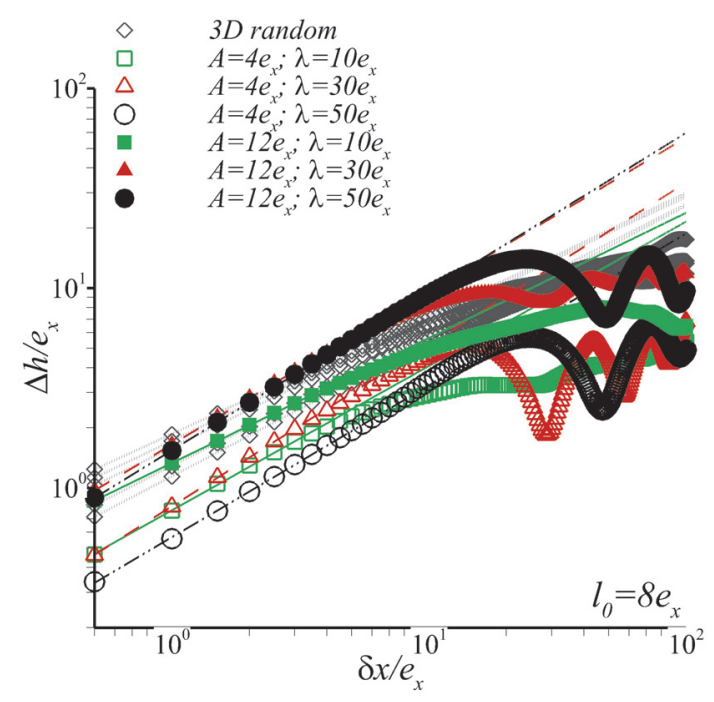

(b)

Figure 9: Height-height correlation functions for two values of amplitude, $A$, and for each $A$ three values of wavelength, $\lambda$, of the sinusoidal distribution of the particles together with five realizations of the 3D random distribution of the particles with mean particle spacing (a) $l_{0}=4 e_{x}$ and (b) $l_{0}=8 e_{x}$.

Both a material's resistance to crack growth and the corresponding fracture surface roughness are strongly affected by its microstructure. Interest in quantitatively relating measures of fracture resistance and measures characterizing fracture surface roughness date back at least to Mandelbrot et al. (1984), see also Bouchaud et al. (1990); Bonamy and Bouchaud (2011). Srivastava et al. (2014) found two regimes of crack growth behavior for random distributions: (i) for sufficiently large particle spacings $\left(l_{0}=8 e_{x}\right.$ is in this regime), crack growth was dominated by a void by void crack growth mode and a linear relation was found between measures of crack growth resistance and fracture surface roughness; while for sufficiently small particle spacings $\left(l_{0}=4 e_{x}\right.$ is in this regime) crack growth was dominated by multiple void interactions and no such relation was found. In both cases, the Hurst exponent characterizing the fracture surface roughness statistics was found to have a universal value. If the crack path follows the sinusoidal particle distribution, the amplitude and wavelength of the sine can be directly related to key fracture surface roughness 
measures. The focus in this Section and, subsequently in Section 5, is: (i) the extent to which the roughness characterizing parameters identified in Srivastava et al. (2014) continue to be key characterizing parameters for controlled inclusion distributions, both when the crack path is sinusoidal and when it differs from the sine; and (ii) the extent to which the toughness-roughness relations found in Srivastava et al. (2014) hold for the controlled particle distributions considered here.

The procedure for fracture surface roughness analysis follows that in Srivastava et al. (2014); Osovski et al. (2015a). Here we briefly describe the procedure, more complete descriptions are given in the references cited. The distributions of void volume fraction $f$ are obtained on ten equally spaced $z=$ constant planes and triangulated using Delauny triangulation. Then, a uniform grid with a constant spacing of $e_{x} / 2 \times e_{x} / 2$ is projected onto the void volume fraction field and the porosity values are interpolated using a cubic interpolation scheme from the points defined by the Delauny triangulation. The interpolation procedure is carried out using Matlab's grid data function. The fracture surface is identified by identifying contours of $f=0.1$ starting from the initial crack tip and extending to a specified value of $\Delta a$. This gives 20 fracture surfaces (10 planes with upper and lower fracture surfaces on each plane). Except for the step of interpolating data from a nonuniform mesh to a uniform grid, this procedure mimics experimentally measuring fracture surface roughness using a profilometer.

Attention is confined to analyzing fracture surface roughness in the direction of crack propagation (the direction parallel to the $x$-axis). The height fluctuations of the fracture surface are characterized by the correlation function, $\Delta h$, given by

$$
\Delta h(\delta x)=\sqrt{\left\langle[h(x+\delta x, z)-h(x, z)]^{2}\right\rangle_{x, z}}
$$

Here, \langle\rangle$_{x, z}$ denotes the average over $x$ and $z$. The quantity $\Delta h(\delta x)$ can be interpreted as the typical difference of height between two points separated by a distance $\delta x$ along the mean fracture plane. The height correlation function is computed for all the 20 planes and then averaged.

Fig. 9 shows log-log plots of the correlation function $\Delta h(\delta x)$ for two values of amplitude, $A=4 e_{x}$ and $12 e_{x}$, and for each $A$ three values of wavelength, $\lambda=10 e_{x}, 30 e_{x}$ and $50 e_{x}$, of the sinusoidal distribution of the particles together with five realizations of the $3 \mathrm{D}$ random distribution of the particles with mean particle spacing $l_{0}=4 e_{x}$ and $8 e_{x}$. A correlation function exhibits power law scaling if

$$
\Delta h(\delta x) \propto \delta x^{\beta}
$$

over a range of values of $\delta x$. In Eq. (17) the exponent $\beta$ is termed the Hurst exponent.

The value of the Hurst exponent $\beta$ lies within the range 0 to 1 . As noted in Osovski et al. (2015a) a function $y(x)=a x+b$ gives a value of $\beta=1$ if $a \neq 0$ and a value $\beta=0$ if $a=0$. We note that these values of $\beta$ are also obtained if small amplitude white noise is superposed on the linear relation. The value $\beta=0.5$ corresponds to a random walk. Values greater than 0.5 indicate persistence, i.e. an increase (decrease) is followed by an increase (decrease) 


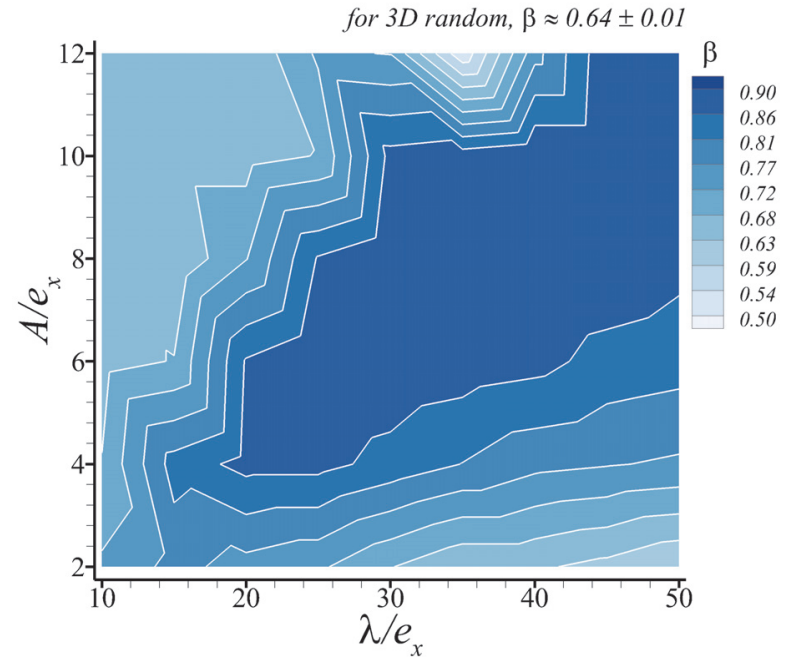

(a)

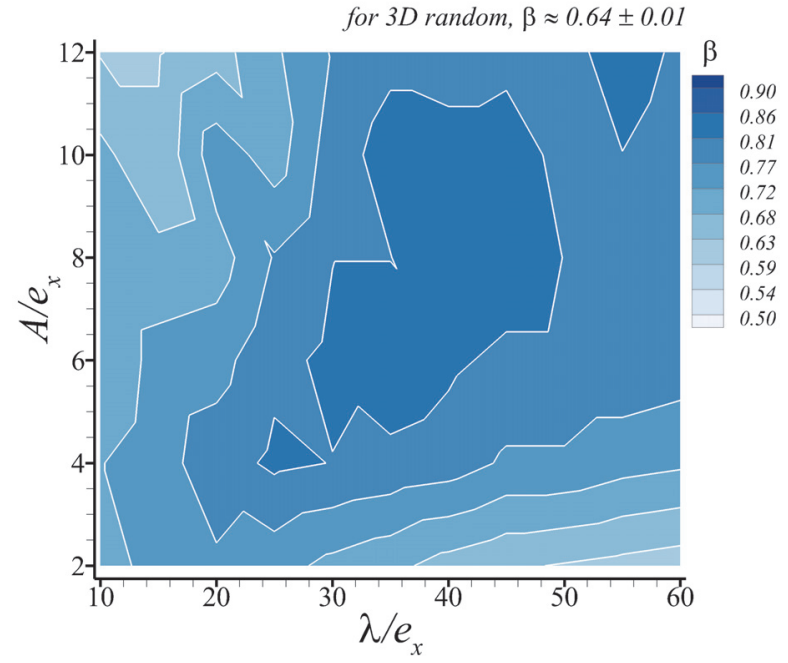

(b)

Figure 10: Variation of the Hurst exponent, $\beta$, with normalized amplitude, $A / e_{x}$, and normalized wavelength, $\lambda / e_{x}$, of the sinusoidal distribution of the particles with mean particle spacing (a) $l_{0}=4 e_{x}$ and (b) $l_{0}=8 e_{x}$.

while $\beta$ less than 0.5 indicate anti-persistence, i.e. an increase (decrease) is followed by a decrease (increase). As seen in Fig. 9, there is a range of $\delta x$ with a good fit to the power law relation given in Eq. (17). The good fit to the power law relation over a range of $\delta x$ in Fig. 9, does not necessarily imply that the underlying statistics are actually power law statistics. Nevertheless, a value of $\beta$ can be calculated and, regardless of the underlying statistics, the computed Hurst exponent $\beta$ serves as a measure of persistence (or anti-persistence).

Here, as illustrated in Fig. 9, the computed height-height correlation functions can be fit to a power law over a range of $\delta x$. The value of the exponent $\beta$ of the power law is calculated as the slope of the dashed line fit to the log-log plot of $\Delta h$ versus $\delta x$ over the range $\delta x<4 e_{x}$. The variation of this small scale $\beta$ with amplitude, $A$, and wavelength, $\lambda$, of the sinusoidal distribution of the particles with mean particle spacing $l_{0}=4 e_{x}$ and $8 e_{x}$ is shown in Fig. 10. Interestingly unlike in Srivastava et al. (2014) where for a random distribution of particles the value of the small scale $\beta$ was found to have a universal value (constant average value of $\beta$ independent of the mean particle spacing), the computed value of $\beta$ for the controlled microstructure does not have a universal value. As seen in Fig. 10, $\beta$ increases when the amplitude $A$ and the wavelength $\lambda$ of the sinusoidal profile increase together. For small amplitudes and small wavelengths, $\beta$ is close to 0.5 whereas for large amplitudes and large wavelengths, $\beta$ approaches unity. On the other hand, the value of $\beta$ for the random distribution of particles is $0.64 \pm 0.01$ for both values of $l_{0}$.

As seen in Fig. 9, the power law behavior of the correlation function only holds up to a critical value of $\delta x$, thereafter the slope of the log-log plot of the correlation function tends to decrease. In Srivastava et al. (2014) a cut-off length $\xi$ was defined as the intersection of $\Delta h(\delta x)=\Delta h_{s}$ (where $\Delta h_{s}$ is the value of $\Delta h$ at which slope of the log-log plot of the 


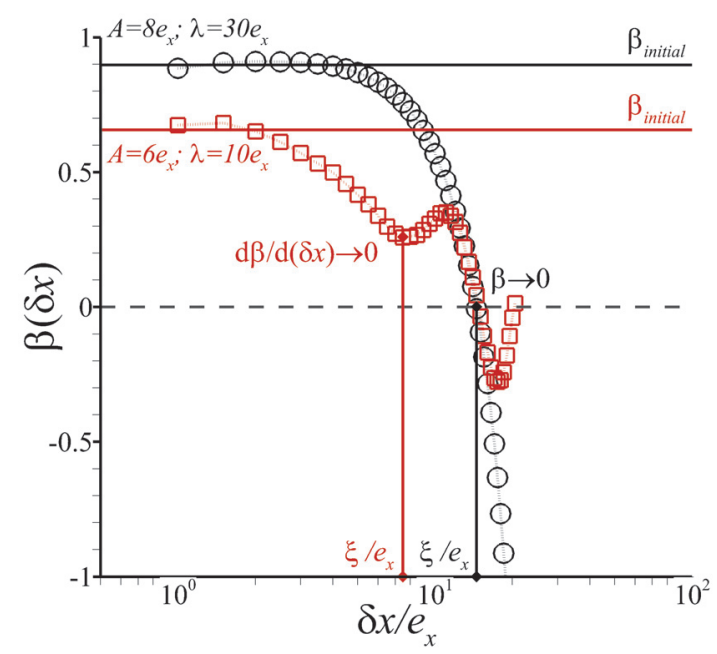

Figure 11: Illustration of the estimation of the cut-off length $\xi$ from two typical variation of the local slope, $\beta(\delta x)$, of the log-log plot of the correlation function. Both the cases shown are for the sinusoidal distribution of particles with mean particle spacing, $l_{0}=4 e_{x}$.

correlation function tends to zero) and the line fit to the initial linear portion of the log-log plot.

The values of the cut-off length $\xi$ and $\Delta h_{s}$ in Srivastava et al. (2014) were found to correlate with measures of crack growth resistance through parameters characterizing the microstructure. In Osovski et al. (2015a) where focus was confined on analyzing the effect of loading rate on the fracture toughness and fracture surface roughness it was found that the slope of the log-log plot of the correlation function beyond the power law regime decreases and tends to zero as in Srivastava et al. (2014). However, in a few cases it was also found that the slope of the log-log plot of the correlation function beyond the power law regime first decreased and then tended to increase. Hence, in Osovski et al. (2015a), $\Delta h_{s}$ was defined as the value of $\Delta h$ at which slope of the log-log plot of the correlation function either tends to zero or as the value of $\Delta h$ at the inflection point at which the log-log plot of the correlation function changes from being concave to convex. The value of $\xi$ was then defined as the intersection of $\Delta h(\delta x)=\Delta h_{s}$ and the line fit to the initial linear portion of the log-log plot.

Here, we define the value of $\xi$ as the value of $\delta x$ at which the slope of the log-log plot of the correlation function either tends to zero or as the value of $\delta x$ at the inflection point at which the slope of the log-log plot of the correlation function changes from being concave to convex, Fig. 11. The value of $\Delta h_{s}$ is then the value of $\Delta h$ at $\delta x=\xi$. This definition of $\Delta h_{s}$ is similar to that in Osovski et al. (2015a) but the definition of $\xi$ differs. Subsequently it will be shown that the definition of $\xi$ here more accurately captures the fracture surface roughness than the value of $\xi$ as defined in Srivastava et al. (2014); Osovski et al. (2015a).

Figs. $12 \mathrm{a}$ and $\mathrm{b}$ show the variation of the normalized cut-off length, $\xi / e_{x}$ (as defined in Fig. 11) with normalized wavelength, $\lambda / e_{x}$, for sinusoidal distributions with mean spacings 


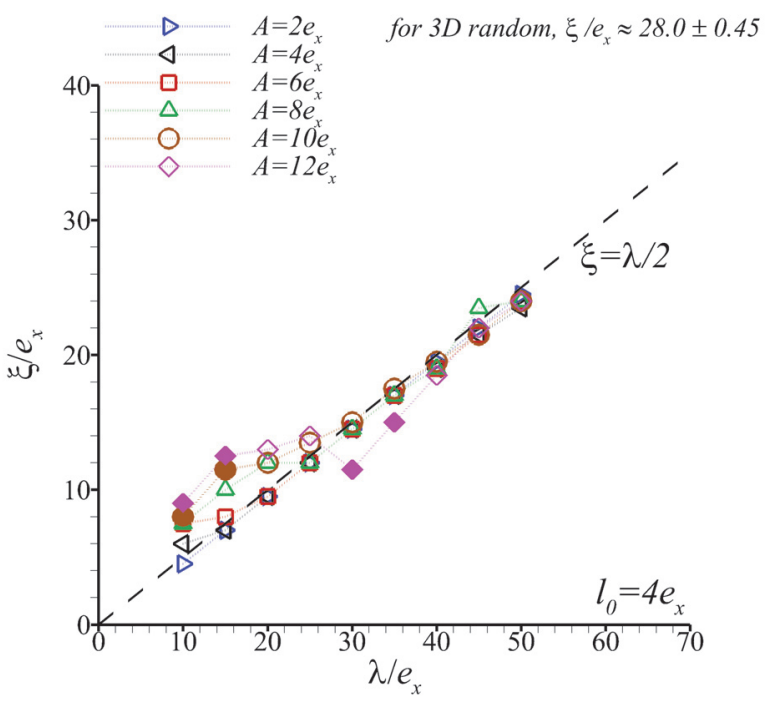

(a)

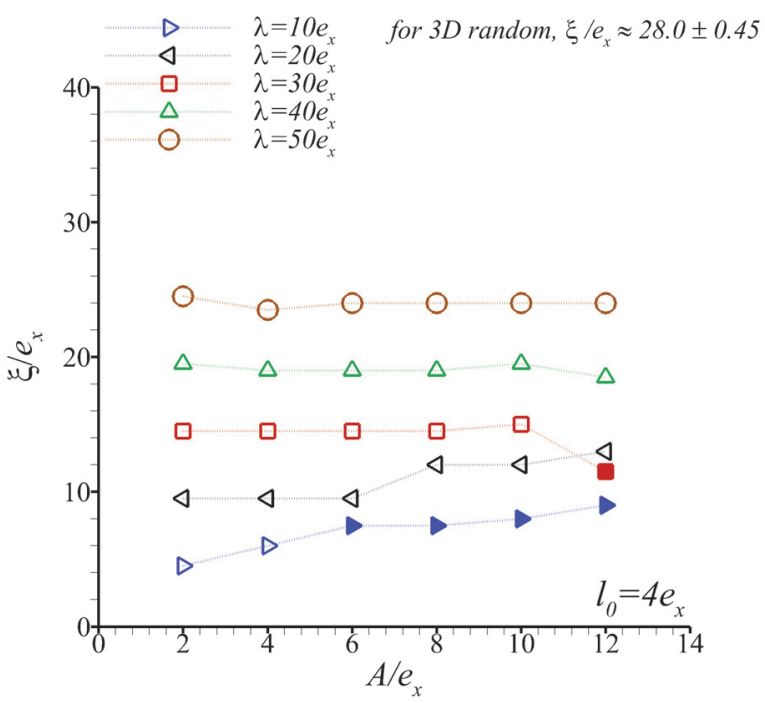

(c)

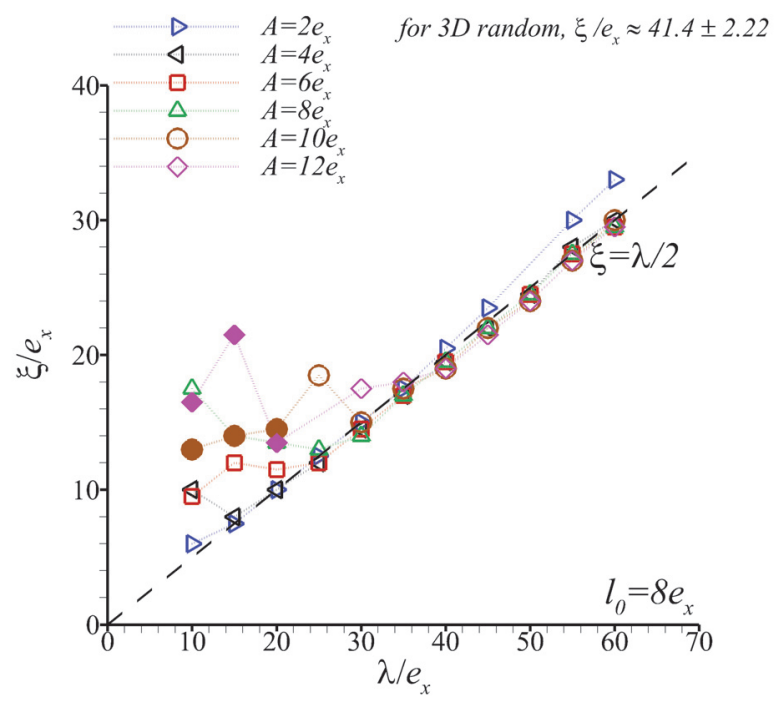

(b)

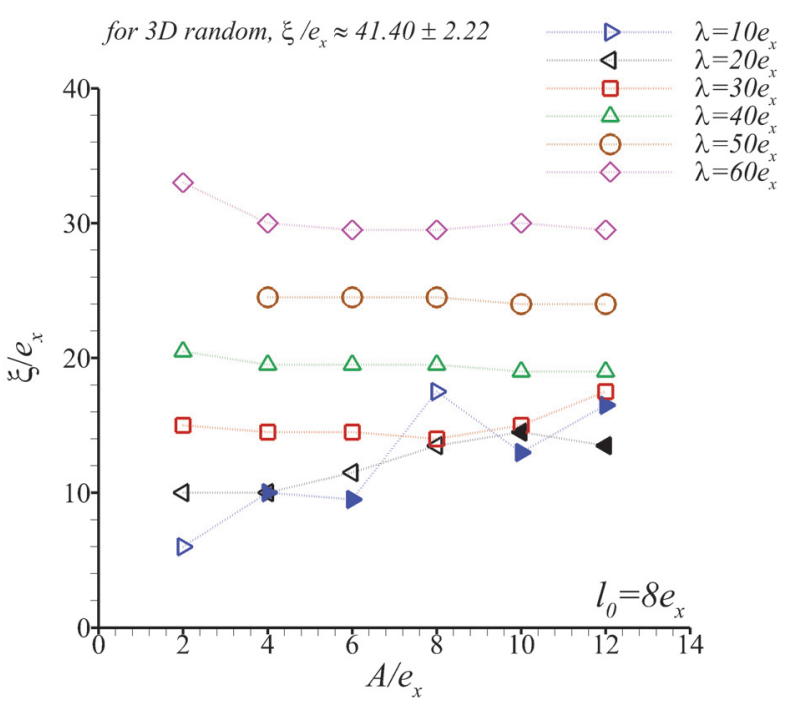

(d)

Figure 12: Variation of normalized cut-off length, $\xi / e_{x}$, with normalized wavelength, $\lambda / e_{x}$, of the sinusoidal distribution of the particles for fixed values of amplitude, $A$, for mean particle spacing (a) $l_{0}=4 e_{x}$ and (b) $l_{0}=8 e_{x}$; and variation of $\xi / e_{x}$ with normalized amplitude, $A / e_{x}$, of the sinusoidal distribution of the particles for fixed values of $\lambda$ for (c) $l_{0}=4 e_{x}$ and (d) $l_{0}=8 e_{x}$.

$l_{0}=4 e_{x}$ and $8 e_{x}$. The corresponding variations of $\xi / e_{x}$ with normalized amplitude $A / e_{x}$ are shown in Figs. 12c and d. The open symbols in Fig. 12 correspond to the values of $\xi$ obtained from $\beta \rightarrow 0$ whereas the filled symbols corresponds to the value of $\xi$ obtained from $d \beta(\delta x) / d(\delta x) \rightarrow 0$, Fig. 11. As seen in the Fig. 12, the value of $\xi$ increases with increasing wavelength $\lambda$, whereas the value of $\xi$ is nearly independent of $A$. 


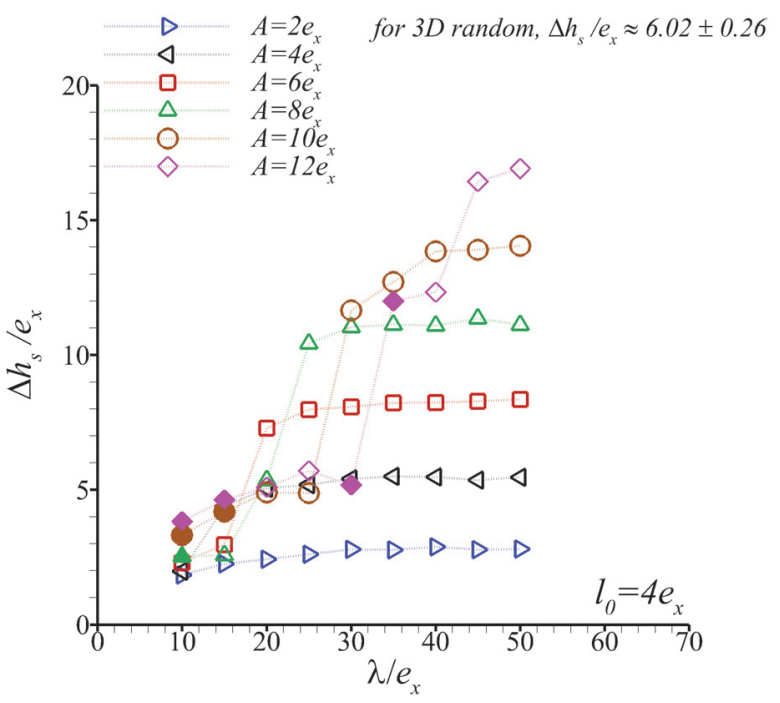

(a)

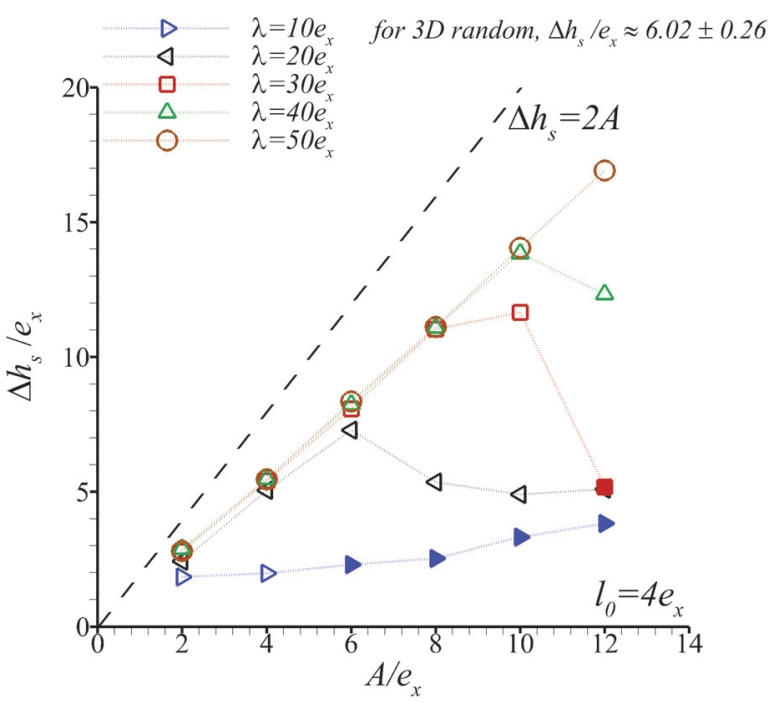

(c)

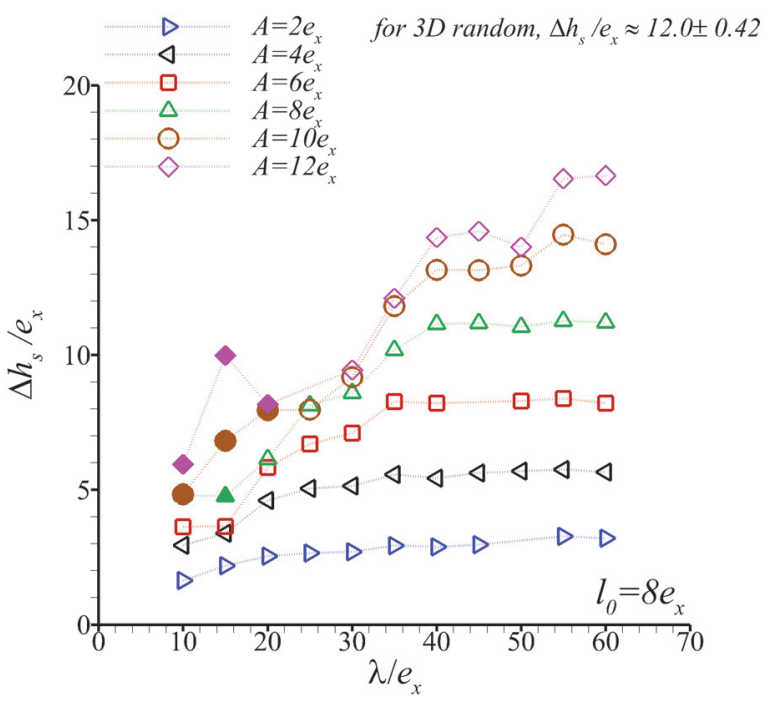

(b)

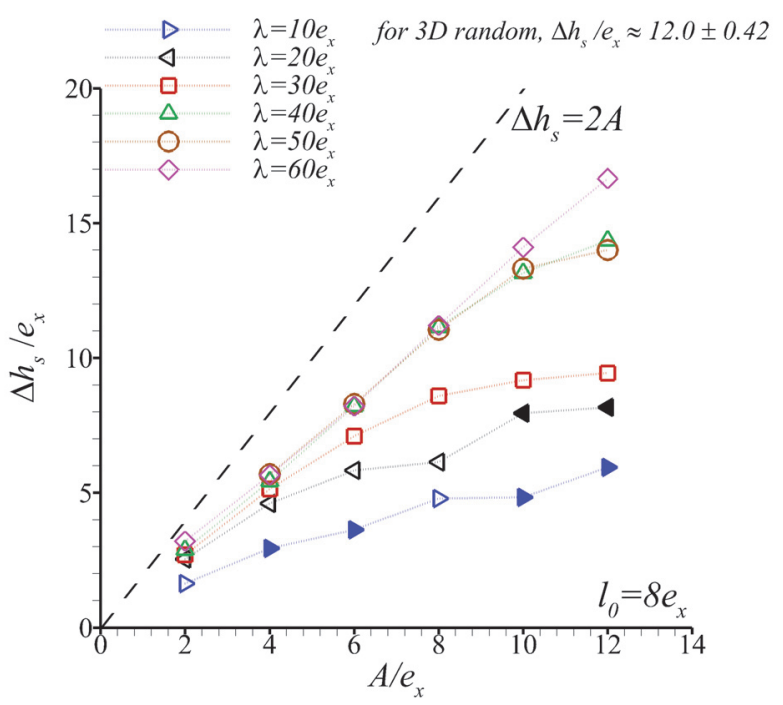

(d)

Figure 13: Variation of normalized saturation value of the correlation function, $\Delta h_{s} / e_{x}$, with normalized wavelength, $\lambda / e_{x}$, of the sinusoidal distribution of the particles for fixed values of amplitude, $A$, for mean particle spacing (a) $l_{0}=4 e_{x}$ and (b) $l_{0}=8 e_{x}$; and variation of $\Delta h_{s} / e_{x}$ with normalized amplitude, $A / e_{x}$, of the sinusoidal distribution of the particles for fixed values of $\lambda$ for (c) $l_{0}=4 e_{x}$ and (d) $l_{0}=8 e_{x}$.

In Srivastava et al. (2014) where attention was confined to random distributions of particles with fixed $l_{0}$, a possible physical description of $\xi$ as the wavelength of the larger zig-zag excursions of the crack path in the microstructure emerged. This suggests that for a controlled microstructure characterized by a sinusoidal distribution of particles, if the crack follows the microstructure, the cut-off length, $\xi$, should be approximately half the wave- 
length of the sinusoidal profile. Indeed, as shown in Figs. $12 \mathrm{a}$ and $\mathrm{b}$, the value of $\xi \approx \lambda / 2$, for both mean particle spacings. There is a slight deviation in the values of $\xi$ from $\xi=\lambda / 2$ for $l_{0}=8 e_{x}$ when the amplitude $A$ is large and the wavelength $\lambda$ is small.

The variation of the normalized saturation value of the correlation function, $\Delta h_{s} / e_{x}$, with normalized wavelength, $\lambda / e_{x}$, for the sinusoidal distribution of the particles with $l_{0}=4 e_{x}$ and $8 e_{x}$ are shown in Figs. 13a and b. Figs. 13c and d show the corresponding variation of $\Delta h_{s} / e_{x}$ with normalized amplitude, $A / e_{x}$. As seen in Figs. 13a and b, the value of $\Delta h_{s}$ increases with an increase in $\lambda$ until a critical value of $\lambda$ is attained and then the value of $\Delta h_{s}$ approaches a plateau. For a fixed mean particle spacing $l_{0}$, this critical value of $\lambda$ depends on the amplitude $A$ and increases with increasing $A$. The plateau level also increases with increasing $A$. Analogous to the relation between $\xi$ and $\lambda, \Delta h_{s}$ should be approximately equal to $2 A$ if the crack follows the controlled microstructure. However, as seen in Figs. 13c and d, $\Delta h_{s}$ in general is below $\Delta h_{s}=2 A$. Nevertheless, the value of $\Delta h_{s}$ does increase with increasing $A$ until a critical value of $A$ is attained and then tends to plateau.

\section{Toughness-roughness correlation}

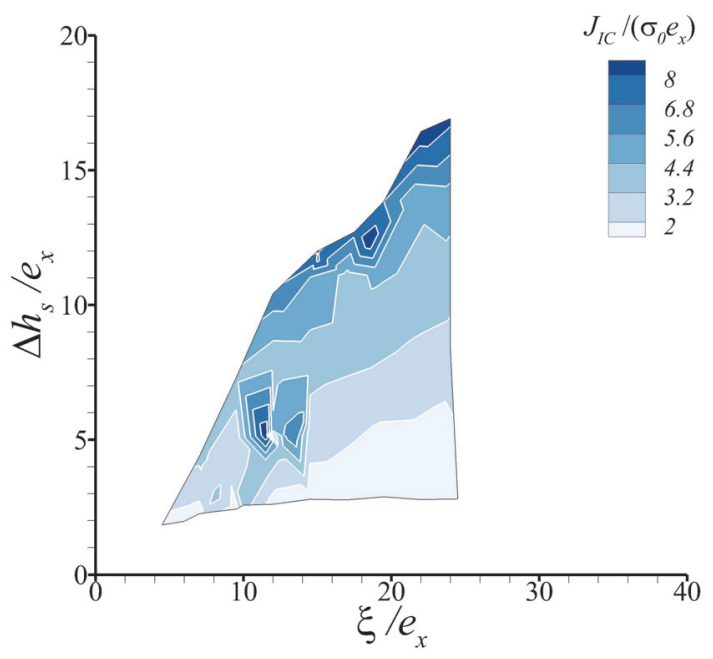

(a)

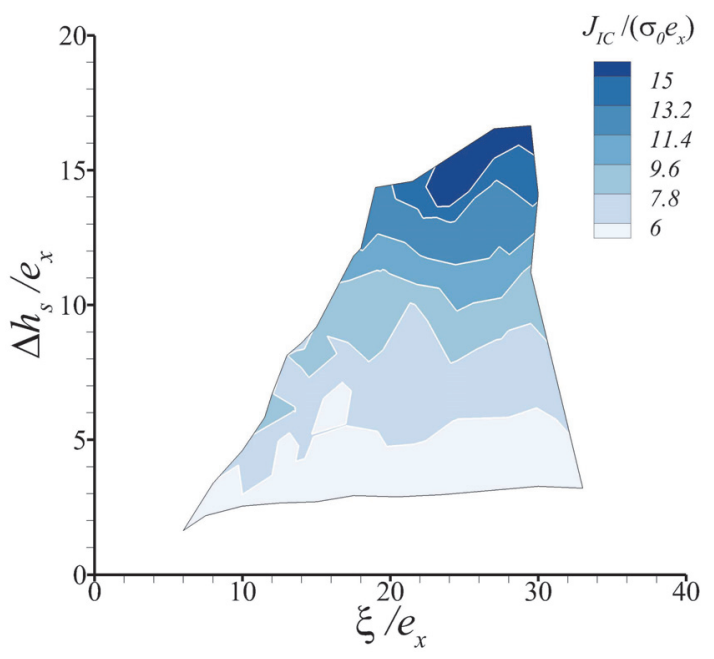

(b)

Figure 14: Variation of normalized fracture toughness, $J_{I C} /\left(\sigma_{0} e_{x}\right)$, with normalized saturation value of the correlation function, $\Delta h_{s} / e_{x}$, and normalized cut-off length, $\xi / e_{x}$, for the sinusoidal distribution of the particles with mean particle spacing (a) $l_{0}=4 e_{x}$ and (b) $l_{0}=8 e_{x}$.

In Srivastava et al. (2014), the parameters characterizing the fracture surface roughness, such as the cut-off length, $\xi$, and the saturation value of the correlation function, $\Delta h_{s}$, were found to be linearly related to the crack growth resistance measures, $J_{I C}$ and $T_{R}$, for microstructures with random distribution of particles for the range of particle volume fractions where the mean particle spacing, $l_{0}$, provided the dominant length scale. Here we explore 


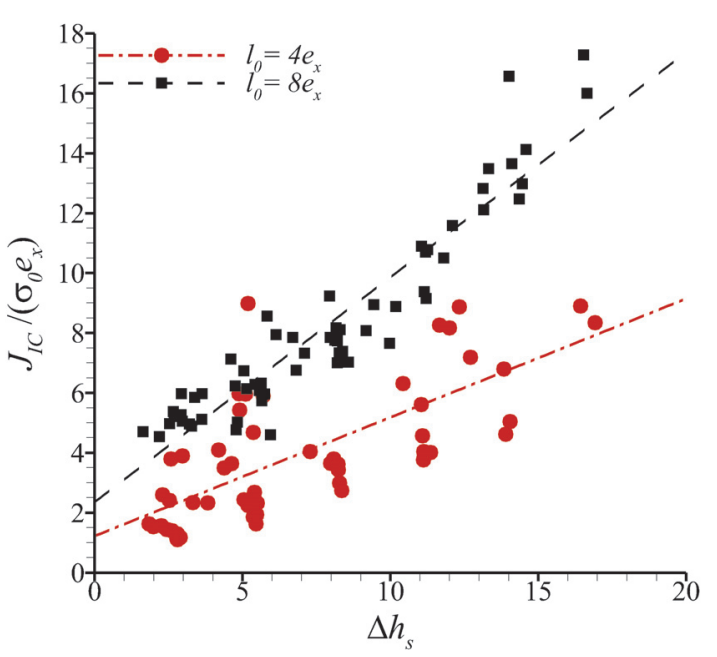

(a)

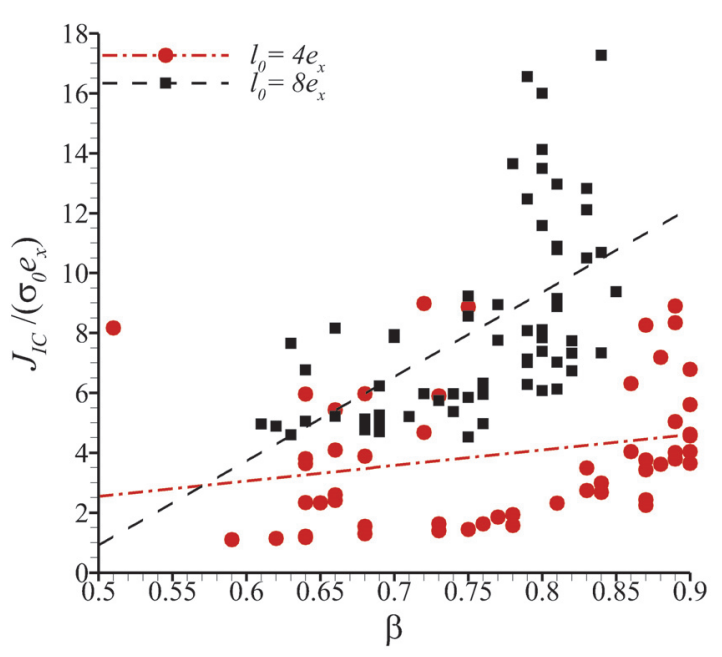

(b)

Figure 15: Variation of normalized fracture toughness, $J_{I C} /\left(\sigma_{0} e_{x}\right)$, with (a) normalized saturation value of the correlation function, $\Delta h_{s} / e_{x}$, and (b) Hurst exponent, $\beta$. The individual plotted points correspond to a combination of amplitude, $A$, and wavelength, $\lambda$, of the sinusoidal distribution with fixed mean particle spacing, $l_{0}$, and the lines are linear fit to the data.

the relation between measures of fracture surface roughness and crack growth resistance for controlled microstructures characterized by the sinusoidal distribution of particles with fixed mean particle spacings. Fig. 14 shows the variation of $J_{I C} /\left(\sigma_{0} e_{x}\right)$ with $\Delta h_{s} / e_{x}$ and $\xi / e_{x}$, for the sinusoidal distributions of particles with mean particle spacings, $l_{0}=4 e_{x}$ and $8 e_{x}$. As seen in Fig. 14, $J_{I C} /\left(\sigma_{0} e_{x}\right)$ increases when both $\Delta h_{s} / e_{x}$ and $\xi / e_{x}$ increase. The effect of $\Delta h_{s} / e_{x}$ on $J_{I C} /\left(\sigma_{0} e_{x}\right)$ is greater than that of $\xi / e_{x}$ for both the values of $l_{0}$.

The variation of $T_{R}$ with $\Delta h_{s} / e_{x}$ and $\xi / e_{x}$ for $l_{0}=4 e_{x}$ and $8 e_{x}$ is shown in the supplementary material. The trend for the dependence of $T_{R}$ on $\Delta h_{s} / e_{x}$ and $\xi / e_{x}$ is similar to that in Fig. 14a for $l_{0}=4 e_{x}$ but no such trend is seen for $l_{0}=8 e_{x}$.

The relation between $J_{I C} /\left(\sigma_{0} e_{x}\right)$ and $\Delta h_{s} / e_{x}$ for the sinusoidal distribution of the particles with $l_{0}=4 e_{x}$ and $8 e_{x}$ is shown in Fig. 15a. A reasonably good quantitative linear fit is obtained for $l_{0}=8 e_{x}$. There is a similar trend for $l_{0}=4 e_{x}$ but with increased scatter. The relation between $J_{I C}$ and $\xi$ is similar to the relation between $J_{I C}$ and the wavelength, $\lambda$, of the sinusoidal profile since $\xi \approx \lambda / 2$. In Srivastava et al. (2014) and Osovski et al. (2015a), for random distributions of particles, in the small scale regime (a scale of the order of a microstructural length scale) the Hurst exponent, $\beta$, was found to be independent of microstructural variations and applied loading rate. However, for the sinusoidal particle distributions $\beta$ varies with variations in amplitude and wavelength of the sinusoidal profile, Fig. 10. The variation of normalized fracture toughness, $J_{I C} /\left(\sigma_{0} e_{x}\right)$, with $\beta$ is shown in Fig. 15b. There is no simple correlation between the value of $\beta$ and the value of $J_{I C} /\left(\sigma_{0} e_{x}\right)$. However, values of $\beta$ greater than $\approx 0.8$ tend to correspond to significantly increased values 
of $J_{I C}$.

\section{Discussion}

The calculations here indicate that by engineering particle distributions a significant gain in crack growth resistance can be obtained. For a given mean particle spacing, an appropriately engineered sinusoidal particle distribution can have a fracture toughness (the $J_{I C}$ value) that is 2 to 3 times greater than that for a random distribution with the same mean particle spacing. For some sinusoidal particle distributions the fracture toughness is less than that of the corresponding random distribution, which indicates that a properly designed controlled particle distribution is needed to increase the fracture toughness. The increases obtained for the values of the tearing modulus (the $T_{R}$ values) are smaller; increasing by a factor of 1.5 to 2 , with the largest increases occurring for the larger mean particle spacing $\left(l_{0}=8 e_{x}\right)$.

A quantitative comparison with experiment requires the development of processing techniques that can provide accurate control over the location of void nucleating particles in metallic alloys. Hopefully, our predictions will provide the motivation to control particle distributions (or, more generally, defect distributions) in metallic alloys to engineer the crack path in order to increase its ductile fracture resistance. In this regard, it is worth noting that Malik and Barthelat (2016) showed experimentally that engineering the crack path along a sinusoidal weak interface in thin sheets of brittle aluminum oxide can increase its fracture toughness under mode I dominant loading. Furthermore, several highly mineralized natural materials contain well architectured defect distributions that can engineer the crack path. Guided by such natural materials Mirkhalaf et al. (2014) architectured the defect distribution in glass using a 3D laser engraving technique and showed that the laser-generated defect distribution can engineer the crack path and enhance fracture toughness.

Here, the crack growth resistance of the sinusoidal particle distribution is found to be nearly independent of wavelength for small values of amplitude and nearly independent of amplitude for small values of wavelength, Fig. 7. For sufficiently large wavelengths, the crack growth resistance increases with increasing amplitude and for sufficiently large amplitude the crack growth resistance increases with increasing wavelength. Interestingly, for a fixed mean particle spacing, an increase in wavelength for a given amplitude results in fewer particles along the sinusoidal profile, whereas an increase in amplitude for a given wavelength results in more particles along the sinusoidal profile, Fig. 2. Hence, our results indicate that the crack growth resistance can be enhanced either by adding void nucleating particles or by removing them. However, if for a given wavelength, the amplitude is increased beyond a critical value, the propensity for the crack to jump from crest to crest or from trough to trough increases. As a consequence the crack path becomes less tortuous and the plastic dissipation accompanying crack growth decreases. On the other hand, if, for a given amplitude, the wavelength is increased beyond a critical value, the deviation of the crack path from the initial crack line decreases so that, in this circumstance also, the plastic dissipation accompanying crack growth decreases. The largest increase in crack growth resistance is found to occur when both the amplitude and wavelength of the sinusoidal 
distributions are increased together while keeping the ratio of the amplitude to quarter wavelength, $A /(\lambda / 4) \approx 1$ (as seen in the supplementary material).

The precise values of amplitude, $A$, and wavelength, $\lambda$, that give the largest increase in crack growth resistance will depend on material properties such as the flow strength, strain hardening and strain rate hardening. In addition, these values will depend on the parameters characterizing void nucleation, growth and coalescence.

In ductile fracture, the largest contribution to the plastic dissipation accompanying crack growth comes from the large scale plastic dissipation outside the fracture process zone. The plastic dissipation associated with the fracture process is generally a small fraction of the plastic dissipation associated with crack growth.

Here, as in Osovski et al. (2015a), the plastic dissipation in the fracture process region or damage zone, $W_{D Z}^{P}$, is obtained by calculating plastic dissipation in regions where $f \geq 0.04$ (since $\bar{f}_{N}^{\text {stress }}=\bar{f}_{N}^{\text {strain }}=0.04$ )

$$
W_{D Z}^{P}=\int_{0}^{t}\left[\int \boldsymbol{\tau}: \mathbf{d}^{p} d V_{(f \geq 0.04)}\right] d t
$$

The expression in Eq. (18) neglects the work associated with creating new free surface but that is expected to be small in comparison with the plastic dissipation accompanying the ductile fracture process.

The total plastic dissipation, $W^{P}$, is given by

$$
W^{P}=\int_{0}^{t}\left[\int \boldsymbol{\tau}: \mathbf{d}^{p} d V\right] d t
$$

In the calculations of Osovski et al. (2015a) the ratio, $W_{D Z}^{P} / W^{P}$ was generally found to lie within a range between 0.05 and 0.25 .

As noted by Rice and Wang (1989) and seen in Osovski et al. (2015a), an increase in the plastic dissipation in the fracture process zone can lead to a greater increase in plastic dissipation outside the process zone. Hence, engineering material microstructure to enhance ductile crack growth resistance also involves consideration of the relation between the fracture process zone plastic dissipation and the plastic dissipation taking place outside the fracture process zone. For the sinusoidal particle distributions analyzed, the variation of total plastic dissipation, $W^{P}$, with plastic dissipation in the damage region, $W_{D Z}^{P}$, is shown in Fig. 16. The results for $l_{0}=4 e_{x}$ at two applied $J$ levels are shown in Figs. 16a and 16b, while Figs. $16 \mathrm{c}$ and $16 \mathrm{~d}$ show results for $l_{0}=8 e_{x}$. The ratio $W_{D Z}^{P} / W^{P}$ lies within the same general range as in Osovski et al. (2015a). For a given mean particle spacing $l_{0}$, increasing $W_{D Z}^{P}$ by varying the values of $A$ and $\lambda$ of the sinusoidal distribution, increases $W^{P}$. There is a good linear fit in all four plots in Fig. 16 and, for a given mean particle spacing $l_{0}$, the slope of the linear fit decreases with increasing applied $J$.

The material response in our dynamic calculations is taken to be rate and temperature dependent with adiabatic conditions assumed to hold. The effect of material density, i.e. the role of inertia, on ductile crack growth resistance and fracture surface roughness predictions was analyzed in Osovski et al. (2015a) where it was found that the overall trends in crack 


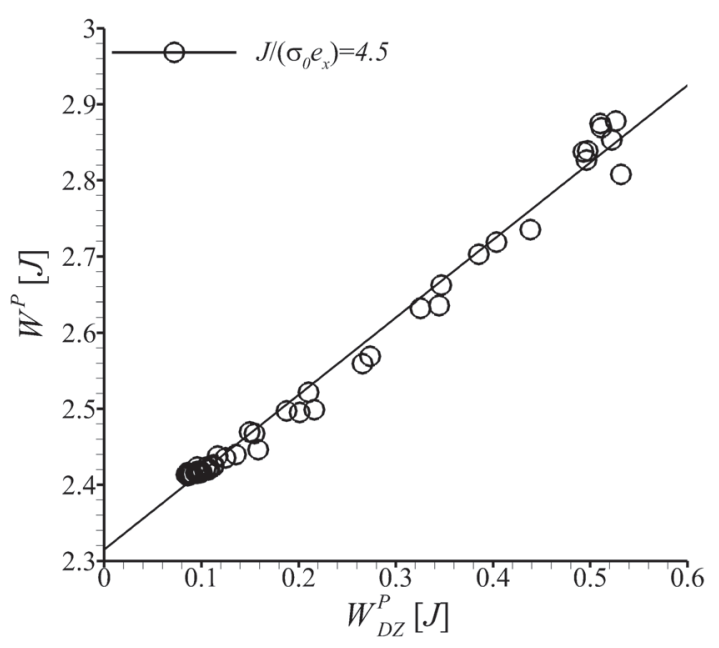

(a)

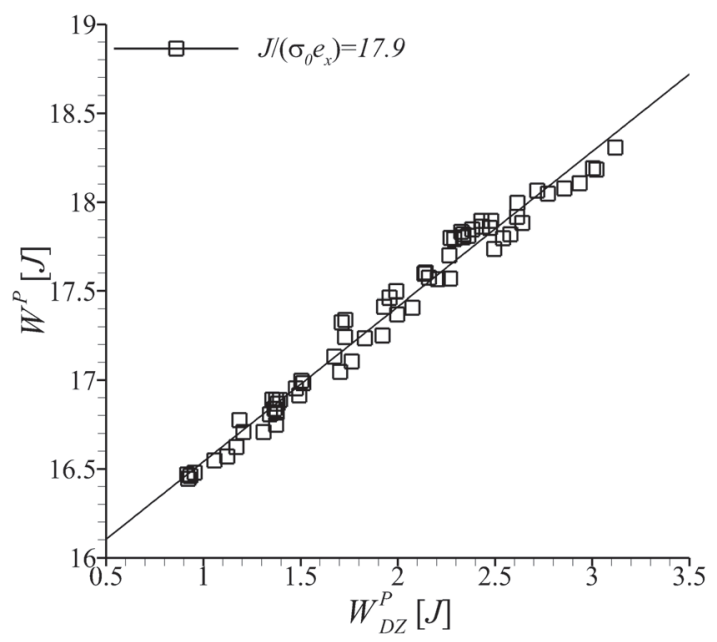

(c)

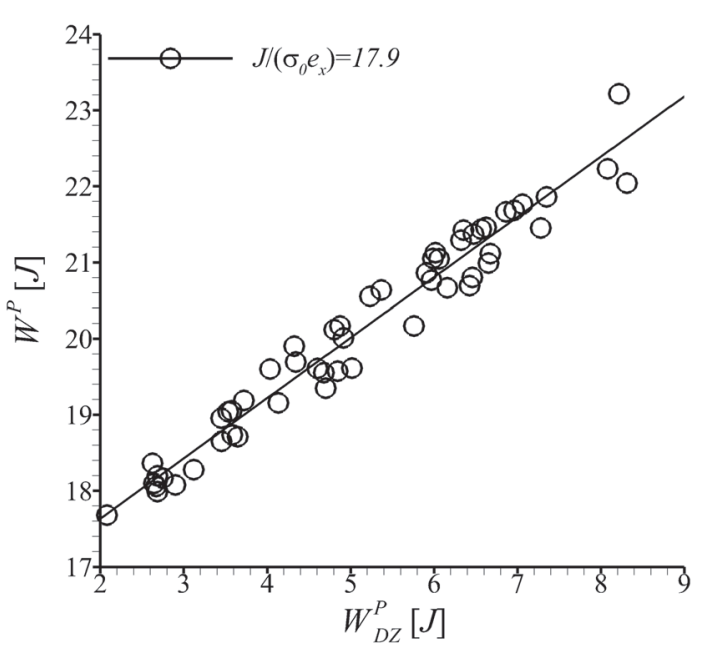

(b)

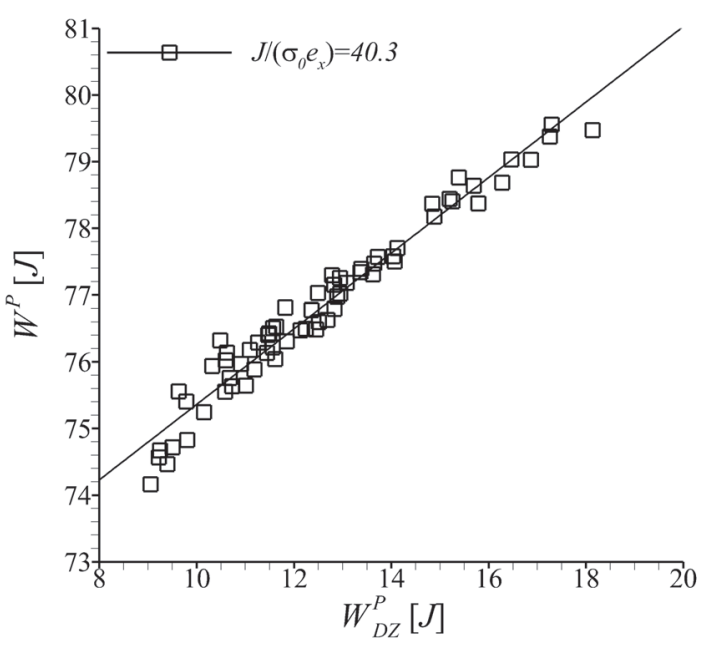

(d)

Figure 16: Variation of total plastic dissipation, $W^{P}$, with plastic dissipation in the damage region, $W_{D Z}^{P}$, for mean particle spacing, $l_{0}=4 e_{x}$ at applied normalized $J$, (a) $J /\left(\sigma_{0} e_{x}\right)=4.5$ and (b) $J /\left(\sigma_{0} e_{x}\right)=17.9$, and for $l_{0}=8 e_{x}$ at, (c) $J /\left(\sigma_{0} e_{x}\right)=17.9$ and $(\mathrm{d}) J /\left(\sigma_{0} e_{x}\right)=40.3$. The $W_{D Z}^{P}$ versus $W^{P}$ data points correspond to a combination of amplitude, $A$, and wavelength, $\lambda$, of the sinusoidal distribution with fixed mean particle spacing, $l_{0}$, and the solid line is linear fit to the data.

growth resistance and fracture surface roughness measures were unaffected by a three fold change in the value of the material density and, for the loading rate in the calculations here, the difference in the quantitative values were found to be less than $10 \%$. Also, the largest temperature increase in our calculations was about $100 \mathrm{~K}$, which corresponds to a decrease in flow strength by approximately 10\%, and occurs within localization bands near failure. 
In Osovski et al. (2015a) the appropriateness of the assumption of adiabatic conditions was also investigated and it was found that during the time a strain gradient develops over a given length scale, the distance over which heat conduction occurred is smaller than this length scale, indicating that the adiabatic assumption is valid for the loading rate used in the calculations here.

Beginning with the work of Mandelbrot et al. (1984) there has been interest in relating the fractal dimension (or Hurst exponent) characterizing the fracture surface roughness to some measure of fracture resistance. However, this has not been successful. Bouchaud et al. (1990) argued that the lack of success stems from the fact that the Hurst exponent characterizing the scale invariance of the fracture surface roughness has a universal value. The Hurst exponent measured along the crack propagation direction is observed to take a value $\beta \approx 0.5$ for brittle failure while for damage accompanying failure $\beta \approx 0.6$ is observed (Ponson et al., 2006; Bonamy and Bouchaud, 2011). Srivastava et al. (2014) argued that the reason for a "universal" value of $\beta>0.5$ (indicating persistence) for ductile fracture can, at least in part, be rationalized by noting that the roughness in a random microstructure is close to a random walk (a Hurst exponent of $1 / 2$ ) but is biased to a larger value by the tendency of cracks (and micro-cracks) to continue growing, at least for a while, in the same direction.

The correlation function, $\Delta h(\delta x)$, in Fig. 9, characterizing the fracture surface roughness in the crack growth direction of the sinusoidal microstructure can be fit to a power law for $\delta x<\xi$. It is worth noting that although the particles are constrained to lie along a sinusoidal profile, within that profile the particle distribution is random. The cut-off size scale for power law behavior, $\xi$, for the sinusoidal particle distributions is linearly related to the half wavelength of the sine, Figs. 12a and b. In addition to the length scales associated with the sinusoidal profile (the amplitude and the wavelength), the mean particle spacing provides a length scale as does the finite element mesh which at least sets a minimum length scale that can appropriately be considered. The latter two length scales are also associated with the random distribution of particles in Srivastava et al. (2014); Osovski et al. (2015a). In Srivastava et al. (2014) and Osovski et al. (2015a), for random distribution of particles, in the small scale regime, $e_{x} / 2 \leq \delta x<4 e_{x}$, (a scale with lower bound set by finite element mesh size and upper bound set by the mean particle spacing) Hurst exponent, $\beta$, was found to be independent of microstructural variations and applied loading rate. The situation is, however, quite different for the sinusoidal distribution of particles. For the sinusoidal distribution of particles the value of the small scale $\beta$ varies in the range, $0.5<\beta<1$, Fig. 10. In general, the value of $\beta$ is found to increase when both the amplitude and the wavelength of the sine increase, which is also true for the fracture toughness, $J_{I C}$. However, no clear correlation between $\beta$ and fracture toughness was obtained, Fig. 15b. Nevertheless, the value of $\beta$ serves as a measure of persistence and our results indicate that simultaneously increasing both amplitude and wavelength of the sinusoidal profile results in increased persistence in crack growth, at least in the small scale regime, leading to increased fracture toughness.

In Srivastava et al. (2014) the parameters characterizing the fracture surface roughness, such as the cut-off size scale for power law behavior, $\xi$, and the corresponding roughness 
amplitude, $\Delta h_{s}$, were found to correlate with a microstructural length scale in the same way as the crack growth resistance measures $J_{I C}$ and $T_{R}$. Thus, in circumstances where the microstructural length scale dominated the crack growth process $\xi$ and $\Delta h_{s}$ were also found to correlate with $J_{I C}$ and $T_{R}$. In Srivastava et al. (2014), a possible physical description of $\xi$ as the wavelength and that of $\Delta h_{s}$ as the amplitude of the larger zig-zag excursions of the crack path in the microstructure emerged. In the calculations here, the resulting fracture surface roughness, at least for the circumstances when the crack follows the controlled sinusoidal particle distribution, provides a clearer physical description of the parameters characterizing the fracture surface roughness. Indeed, as shown in Figs. 12 and 13, $\xi$ and $\Delta h_{s}$ correspond to the wavelength and amplitude, respectively, of the zig-zag excursions of the crack path. The wavelengths and amplitudes of the zig-zag excursions of the fracture surface roughness relate to the wavelengths and amplitudes of the sinusoidal particle distributions in the circumstances where the crack follows the microstructure. Hence, in these circumstances, where microstructural features/length scales dictate the crack path, there is a correlation between parameters characterizing the fracture surface roughness and the ductile crack growth resistance.

\section{Conclusions}

We have analyzed crack growth under mode I small scale yielding conditions for controlled microstructures characterized by sinusoidal distribution of large second phase particles that nucleate voids relatively early in the deformation history and a uniform distribution of smaller particles that nucleate voids at much larger strains. Calculations were carried out for two values of mean particle spacing and for various values of amplitude and wavelength of the sinusoidal distributions.

1. For the range of microstructural parameters considered here the value of $J_{I C}$ for an appropriately engineered sinusoidal distribution of particles can be 2 to 3 times greater than that for a random distribution of particles with the same mean particle spacing.

1a. The value of $J_{I C}$ is found to be nearly independent of wavelength for small values of amplitude and is nearly independent of amplitude for small values of wavelength.

1b. The value of $J_{I C}$ for sufficiently large wavelength increases with increasing amplitude and for sufficiently large amplitude increases with increasing wavelength.

1c. The increase in the value of $J_{I C}$ is generally greatest when both amplitude and wavelength are large.

2. For the range of microstructural parameters considered here the maximum tearing modulus, $T_{R}$, for sinusoidal distributions of particles can be 1.5 to 2 times greater than that for a random distribution of particles with the same mean particle spacing. But the value of $T_{R}$ is generally less sensitive than the value of $J_{I C}$ to the amplitude and wavelength of the sinusoidal particle distribution, at least in the circumstances analyzed. 
3. If for a given wavelength, the amplitude is increased beyond a critical value, the crack path does not follow the particle distribution and can jump from one peak to the next or from one trough to the next. On the other hand, for a given amplitude, if the wavelength is increased beyond a critical value, the overall deviation of the crack path from the initial crack line decreases significantly. Both of these circumstances can result in a decrease in plastic dissipation accompanying crack growth.

4. The small scale (a scale up to the order of the mean particle spacing) value of the computed Hurst exponent $(\beta)$ for the sinusoidal distribution of particles varies in the range, $0.5<\beta<1$. The largest values of $\beta$ are obtained when both the amplitude and the wavelength are large, indicating strong persistence of the crack path in those circumstances.

5. The characteristic length scales of the fracture surface roughness, the cut-off size scale for power law behavior, $\xi$, and the corresponding roughness amplitude, $\Delta h_{s}$, are found to correspond to the wavelength and amplitude of the zig-zag excursions of the crack path, respectively, and, when the crack path follows the sinusoidal profile, $\xi$ and $\Delta h_{s}$ are directly related to the wavelength and amplitude of the sinusoidal profile.

\section{Acknowledgments}

The financial support provided by the U.S. National Science Foundation, Grant CMMI1200203 is gratefully acknowledged. The large-scale finite element calculations reported on were carried out using high performance research computing resources provided by Texas A\&M University (http://hprc.tamu.edu).

\section{References}

ASTM E1820-11, 2011. Standard test method for measurement of fracture toughness, ASTM International. Belytschko T., Chiapetta R.L., Bartel H.D., 1976. Efficient large scale non-linear transient analysis by finite elements. International Journal for Numerical Methods in Engineering, 10, 579-596.

Benzerga A.A., Leblond J.B., 2010. Ductile fracture by void growth to coalescence. Advances in Applied Mechanics, 44, 169-305.

Benzerga A.A., Leblond J.B., Needleman, A., Tvergaard, V., 2016. Ductile failure modeling. International Journal of Fracture, DOI 10.1007/s10704-016-0142-6.

Bonamy D., Bouchaud E., 2011. Failure of heterogeneous materials: A dynamic phase transition?. Physics Reports, 498, 1-44.

Bouchaud, E., Lapasset, G., Planes, J., 1990. Fractal dimension of fractured surfaces: a universal value?. Europhysics Letters, 13, 7379.

Cordisco, F.A., Zavattieri, P.D., Hector, L.G., Bower, A.F., 2012. Toughness of a patterned interface between two elastically dissimilar solids. Engineering Fracture Mechanics, 96, 192-208.

Cordisco, F., Zavattieri, P.D., Hector, L.G., Bower, A.F., 2014. On the mechanics of sinusoidal interfaces between dissimilar elasticplastic solids subject to dominant mode I. Engineering Fracture Mechanics, 131, 38-57.

Chu C.C., Needleman A., 1980. Void nucleation effects in biaxially stretched sheets. Journal of Engineering Materials and Technology, 102, 249-256. 
Frazier W.E., 2014. Metal Additive Manufacturing: A Review. Journal of Materials Engineering and Performance, 23, 1917-1928.

Fullwood D.T., Niezgoda S.R., Adams B.L., Kalidindi S.R., 2010. Microstructure sensitive design for performance optimization. Progress in Materials Science, 55, 477-562.

Garrison Jr W.M., Moody N.R., 1987. Ductile fracture. Journal of Physics and Chemistry of Solids, 48, 1035-1074.

Goods S.H., Brown. L.M., 1979. The nucleation of cavities by plastic deformation. Acta Metallurgica, 27, $1-15$.

Gurland J., Plateau J., 1963. The mechanism of ductile rupture of metals containing particles. Transactions of ASM, 56, 442-454.

Gurson A.L., 1975. Plastic flow and fracture behavior of ductile materials incorporating void nucleation, growth and interaction. Ph.D. thesis, Brown University, Providence, RI.

Hao S., Moran B., Liu W.K., Olson G.B., 2003. A hierarchical multi-physics model for design of high toughness steels. Journal of Computer-Aided Materials Design, 10, 99-142.

Hao S., Liu W.K., Moran B., Vernerey F., Olson G.B., 2004. Multi-scale constitutive model and computational framework for the design of ultra-high strength, high toughness steels. Computer Methods in Applied Mechanics and Engineering, 193, 1865-908.

Hayes R.L., Ortiz M., Carter E.A., 2004. Universal binding-energy relation for crystals that accounts for surface relaxation. Physical Review B, 69, 172104.

Herderick E., 2011. Additive Manufacturing of Metals: A Review. Proceedings of MS\&T'11, Additive Manufacturing of Metals, Columbus, $\mathrm{OH}$.

Kahziz, M, Morgeneyer, T.F, Maziére, M., HelfenL., Bouaziz, O., Maire, E., 2016. In situ 3D synchrotron laminography assessment of edge fracture in dual-phase steels: quantitative and numerical analysis. Experimental Mechanics, 56, 177-195.

Kulkarni A.J., Krishnamurthy K., Deshmukh S,. Mishra R., 2004. Microstructural optimization of alloys using a genetic algorithm. Materials Science and Engineering: A, 372, 213-20.

Lautridou J., Pineau A., 1981. Crack initiation and stable crack growth resistance in A508 steels in relation to particle distribution. Engineering Fracture Mechanics, 15, 55-71.

Malik, I.A., Barthelat, F., 2016. Toughening of thin ceramic plates using bioinspired surface patterns. International Journal of Solids and Structures, 97, 389-399.

Mandelbrot, B.B., Passoja, D.E., Paullay, A.J., 1984. Fractal character of fracture surfaces of metals. Nature, 308,721722 .

Martin J.W., 1998. Precipitation hardening. 2nd ed. Oxford, Boston: Butterworth-Heinemann.

Mathur K., Needleman A., Tvergaard V., 1996. Three dimensional analysis of dynamic ductile crack growth in a thin plate. Journal of the Mechanics and Physics of Solids, 44, 439-64.

McDowell D.L., 2007. Simulation-assisted materials design for the concurrent design of materials and products. JOM, 59, 21-5.

McDowell D.L., Olson G.B., 2009. Concurrent design of hierarchical materials and structures. Scientific Modeling and Simulations: Springer Netherlands, 207-40.

Mirkhalaf, M., Khayer Dastjerdi, A., Barthelat, F., 2014. Overcoming the brittleness of glass through bioinspiration and micro-architecture. Nature Communications, 5, 3166.

Needleman A., Tvergaard V., 1987. An analysis of ductile rupture modes at a crack tip. Journal of the Mechanics and Physics of Solids, 35, 151-83.

Needleman A., Tvergaard V., Bouchaud E., 2012. Prediction of ductile fracture surface roughness scaling. Journal of Applied Mechanics, 79, 031015.

Olson G.B., 1997. Computational design of hierarchically structured materials. Science, 277, 1237-42.

Osovski S., Srivastava A., Ponson L., Bouchaud E., Tvergaard V., Ravi-Chandar K., Needleman A., 2015a. The effect of loading rate on ductile fracture toughness and fracture surface roughness. Journal of the Mechanics and Physics of Solids, 76, 20-46.

Osovski S., Srivastava A., Williams J.C., Needleman A., 2015b. Grain boundary crack growth in metastable titanium $\beta$ alloys. Acta Materialia, 82, 167-178. 
Pan J., Saje M., Needleman A., 1983. Localization of deformation in rate sensitive porous plastic solids. International Journal of Fracture, 21, 261-278.

Paris P.C., Tada H., Zahoor A., Ernst H., 1979. The theory of instability of the tearing modes in elasticplastic crack growth. Elastic-Plastic Fracture, ASTM STP 668, J.D. Landes, J.A. Begley and G.A. Clarke Eds, American Society for Testing and Materials, 5-36.

Peirce D., Shih C.F., Needleman A., 1984. A tangent modulus method for rate dependent solids. Computer \& Structures, 18, 875-887.

Pineau A., Benzerga A.A., Pardoen T., 2016. Failure of metals I. brittle and ductile fracture. Acta Materialia, 107, 424-483.

Ponson, L., Auradou, H., Vie, P. and Hulin, J.P., 2006. Low self-affine exponents of fractured glass ceramics surfaces. Physical review letters, 97, 125501.

Ponson L., Cao Y., Bouchaud E., Tvergaard V., Needleman A., 2013. Statistics of ductile fracture surfaces: the effect of material parameters. International Journal of Fracture, 184, 137-149.

Puttick K.E., 1959. Ductile fracture in metals. Philosophical Magazine, 4, 964-969.

Rice J.R., 1968. A path-independant integral and the approximate analysis of strain concentration by notches and cracks. Journal of Applied Mechanics, 35, 379-386.

Rice J.R., Wang, J.-S., 1989. Embrittlement of interfaces by solute segregation. Materials Science and Engineering, A107, 23-40.

Rogers H.C., 1960. The tensile fracture of ductile metals. AIME TRANS, 218, 498-506.

Srivastava A., Ponson L., Osovski S., Bouchaud E., Tvergaard V., Needleman A., 2014. Effect of inclusion density on ductile fracture toughness and roughness. Journal of the Mechanics and Physics of Solids, 63, 62-79.

Tang S., Kopacz A.M,. Chan S., Olson G.B., Liu W.K., 2013. Three-dimensional ductile fracture analysis with a hybrid multiresolution approach and microtomography. Journal of the Mechanics and Physics of Solids, 61, 2108-24.

Tipper C.F., 1949. The fracture of metals. Metallurgia, 33, 133-157.

Tvergaard V., 1990. Material failure by void growth to coalescence. Advances in Applied Mechanics, 27, 83-151.

Tvergaard V., 1981. Influence of voids on shear band instabilities under plane strain conditions. International Journal of Fracture, 17, 389-407.

Tvergaard V., 1982a. On localization in ductile materials containing spherical voids. International Journal of Fracture, 18, 237-252.

Tvergaard V., 1982b. Influence of void nucleation on ductile shear fracture at a free surface. Journal of the Mechanics and Physics of Solids, 30, 399-425.

Tvergaard V., Hutchinson J.W., 2002. Two mechanisms of ductile fracture: void by void growth versus multiple void interaction. International Journal of Solids and Structures, 39, 3581-3597.

Tvergaard V., Needleman A., 1984. Analysis of the cup-cone fracture in a round tensile bar. Acta Metallurgica, $32,157-169$.

Tvergaard V., Needleman A., 2006. Three dimensional microstructural effects on plane strain ductile crack growth. International journal of solids and structures, 43, 6165-79.

Uchic, M.D., Holzer, L., Inkson, B.J., Principe, E.L., Munroe, P., 2007. Three-dimensional microstructural characterization using focused ion beam tomography. Mrs Bulletin, 32, 408-416.

Ueda, T., Helfen, L., Morgeneyer, T., 2014. In situ laminography study of three-dimensional individual void shape evolution at crack initiation and comparison with GursonTvergaardNeedleman-type simulations. Acta Materialia, 78, 254-270.

Van der Ven A., Ceder G., 2004. The thermodynamics of decohesion. Acta Materialia, 52, 1223-35.

Zavattieri, P.D., Hector Jr, L.G., Bower, A.F., 2007. Determination of the effective mode-I toughness of a sinusoidal interface between two elastic solids. International Journal of Fracture, 145, 167-180.

Zavattieri, P.D., Hector, L.G., Bower, A.F., 2008. Cohesive zone simulations of crack growth along a rough interface between two elasticplastic solids. Engineering Fracture Mechanics, 75, 4309-4332. 